\title{
Phase center modeling for LEO GPS receiver antennas and its impact on precise orbit determination
}

\author{
Adrian Jäggi - R. Dach • O. Montenbruck • \\ U. Hugentobler - H. Bock - G. Beutler
}

Received: 25 February 2009 / Accepted: 30 June 2009 / Published online: 29 July 2009

(C) Springer-Verlag 2009

\begin{abstract}
Most satellites in a low-Earth orbit (LEO) with demanding requirements on precise orbit determination (POD) are equipped with on-board receivers to collect the observations from Global Navigation Satellite systems (GNSS), such as the Global Positioning System (GPS). Limiting factors for LEO POD are nowadays mainly encountered with the modeling of the carrier phase observations, where a precise knowledge of the phase center location of the GNSS antennas is a prerequisite for high-precision orbit analyses. Since 5 November 2006 (GPS week 1400), absolute instead of relative values for the phase center location of GNSS receiver and transmitter antennas are adopted in the processing standards of the International GNSS Service (IGS). The absolute phase center modeling is based on robot calibrations for a number of terrestrial receiver antennas, whereas compatible antenna models were subsequently derived for the remaining terrestrial receiver antennas by conversion (from relative corrections), and for the GNSS transmitter antennas by estimation. However, consistent receiver antenna models for space missions such as GRACE and TerraSAR-X, which are equipped with non-geodetic receiver antennas, are only available since a short time from robot calibrations. We use GPS data of the aforementioned LEOs of the year 2007
\end{abstract}

\footnotetext{
A. Jäggi $(\varangle) \cdot$ R. Dach · H. Bock · G. Beutler

Astronomical Institute, University of Bern,

Sidlerstrasse 5, 3012 Bern, Switzerland

e-mail: adrian.jaeggi@aiub.unibe.ch

O. Montenbruck

German Space Operations Center,

Deutsches Zentrum für Luft- und Raumfahrt,

82230 Weßling, Germany

\section{U. Hugentobler}

Institut für Astronomische und Physikalische Geodäsie,

Technische Universität München, Arcisstraße 21,

80333 München, Germany
}

together with the absolute antenna modeling to assess the presently achieved accuracy from state-of-the-art reduceddynamic LEO POD strategies for absolute and relative navigation. Near-field multipath and cross-talk with active GPS occultation antennas turn out to be important and significant sources for systematic carrier phase measurement errors that are encountered in the actual spacecraft environments. We assess different methodologies for the in-flight determination of empirical phase pattern corrections for LEO receiver antennas and discuss their impact on POD. By means of independent K-band measurements, we show that zero-difference GRACE orbits can be significantly improved from about 10 to $6 \mathrm{~mm} \mathrm{~K}$-band standard deviation when taking empirical phase corrections into account, and assess the impact of the corrections on precise baseline estimates and further applications such as gravity field recovery from kinematic LEO positions.

Keywords Low-Earth orbiter (LEO) - Precise orbit determination (POD) - Antenna phase center variation (PCV) modeling · Pseudo-stochastic orbit modeling · GPS

\section{Introduction}

In the last decade precise orbit determination (POD) based on observations from global navigation satellite systems (GNSS), such as the Global Positioning System (GPS), has been established as one of the standard methods to derive trajectories of satellites in the low-Earth orbit (LEO) with demanding accuracy requirements. The first extensive use of the GPS for LEO POD was made by the TOPEX/Poseidon altimeter mission to determine the ocean topography (Fu et al. 1994). The analysis of continuously collected GPS carrier phase observations allowed for an orbit determination 
with an accuracy of better than $3 \mathrm{~cm}$ in the radial direction (Bertiger et al. 1994). Since that time the quality of GPSderived LEO trajectories has steadily improved thanks to numerous improvements in the GPS orbit and clock products provided by the International GNSS Service (IGS, Dow et al. 2005), in the dynamic background models (Flechtner et al. 2006), and in modeling the carrier phase observations. For the TOPEX/Poseidon follow-on altimeter mission JASON-1, e.g., radial accuracies of about $1 \mathrm{~cm}$ have been demonstrated thanks to an in-flight calibration of the LEO POD antenna (Haines et al. 2004).

Since the launch of the CHAllenging Minisatellite Payload mission (CHAMP, Reigber et al. 1998) GPS sensors are recognized not only as a key tracking system for POD but also for extracting geophysically relevant information such as the long wavelength part of the Earth's static gravity field (Reigber et al. 2003). Current gravity field recovery missions such as the Gravity Recovery And Climate Experiment (GRACE, Tapley et al. 2004) and the Gravity field and steady-state Ocean Circulation Explorer (GOCE, Drinkwater et al. 2006) also use the GPS high-low satellite-to-satellite tracking (hl-SST) to support the determination of the low degree spherical harmonic coefficients of the Earth's gravity field. In the case of GOCE, these coefficients are exclusively determined from GPS data as the measurements of the core instrument, the three-axis gravity gradiometer, are band-limited (Pail et al. 2006).

Most of the geodetic LEO missions are equipped with a BlackJack GPS receiver, e.g., the CHAMP and GRACE missions, or with its commercial version called Integrated GPS and Occultation Receiver (IGOR), e.g., the TerraSAR-X (Buckreuss et al. 2003) and the Constellation Observing System for Meteorology, Ionosphere, and Climate (COSMIC, Wu et al. 2005) missions. This receiver exhibits a representative noise level of $1 \mathrm{~mm}$ for the $L_{1}$ and $L_{2}$ GPS carrier phase measurements or, equivalently, $2-3 \mathrm{~mm}$ for the ionospherefree linear combination $L_{3}$ (Montenbruck et al. 2006). In order to fully exploit this accuracy for LEO POD, a precise modeling of the antenna phase center location is mandatory. This is particularly true for kinematic solutions which are free of any constraints imposed by models describing the LEO dynamics (Švehla and Rothacher 2005), but holds to a large extent for reduced-dynamic orbits as well. The two GRACE satellites provide an ideal platform to assess the presently achieved accuracy of state-of-the-art LEO orbits derived from GPS hl-SST data. Both satellites are equipped with laser retro-reflector arrays, which allow for an independent validation of the orbit quality with satellite laser ranging (SLR) observations. In addition, the K-band ranging (KBR) system provides the unique opportunity to validate the distance variation between the two GRACE satellites with the ultra-precise K-band range measurements (Dunn et al. 2003).
There are many studies dealing with GRACE POD based on GPS tracking data (e.g., Kroes et al. 2005; Kang et al. 2006; Jäggi et al. 2007; van Helleputte and Visser 2008). All of them neglected, however, antenna phase center variations (PCVs) and adopted the constant offset values of the relative antenna model for the LEO receiver and the GPS transmitter antennas. With the introduction of absolute values for the phase center location in the processing standards of the IGS and the availability of calibrated ground station receiver and GPS transmitter antenna patterns since 5 November 2006 (e.g., Schmid et al. 2005, 2007), improved GPS orbit and clock products are available and enable a refined modeling of the GPS carrier phase measurements. Consistent receiver antenna models are, however, not yet available for all space missions as they are often equipped with non-geodetic antennas that are not in use in the IGS ground tracking network. Triggered by the preparation of the TerraSAR-X mission, a consistent (nominal) antenna model obtained from a robotic measurement system in a field campaign has recently been made available for the antenna/chokering combination deployed on several space missions such as CHAMP, GRACE, and TerraSAR-X (Montenbruck et al. 2009). Such a nominal antenna model, however, does not reflect the influence of error sources which are additionally encountered in the actual spacecraft environment, e.g., the influence of near-field multipath.

Subsequently we mainly focus on GRACE GPS data of the year 2007 to derive precise satellite trajectories in the low-Earth orbit and assess the impact of the LEO receiver antenna model from the ground calibration. We show that additional in-flight calibrations of LEO receiver antennas are indispensable when striving for LEO orbits of highest quality and discuss the impact of unmodeled systematic errors on the LEO trajectories. We present two different approaches to derive empirical phase center correction models, the so-called residual approach and the direct approach, and discuss the limitations of the residual approach with respect to the more refined direct approach.

Section 2 shortly reviews the methods of LEO POD. Section 3 focuses on the phase center modeling of GPS antennas and introduces the residual approach and the direct approach for empirically calibrating LEO receiver antennas in-flight. A detailed study is performed in Sect. 4 to analyze the pros and cons of the two approaches in a simulation environment. Based on real data, Sect. 5 illustrates, together with Sect. 6, the necessity for an empirical in-flight calibration of the GRACE POD antennas to derive GRACE orbits and baselines of highest quality. Section 7 finally shows that the in-flight calibration of LEO receiver antennas is not only relevant for POD but also for geophysical applications such as the extraction of the Earth's gravity field parameters from kinematic GRACE positions. 


\section{LEO orbit determination}

This section describes relevant theoretical aspects for the determination of LEO orbits from GPS hl-SST data as they are implemented in two software packages. The majority of the solutions in this study has been produced with a special version of the Bernese GPS Software (Dach et al. 2007) according to a procedure described by Jäggi et al. (2006). The same software version and processing procedure is currently used at the Astronomical Institute of the University of Bern (AIUB) to derive the GOCE precise science orbit product (Bock et al. 2007) in the context of the High-level Processing Facility of the European GOCE Gravity Consortium (Koop et al. 2006).

For a cross-validation of the LEO orbits generated in this study, in particular of the baseline solutions in Sect. 6, independent solutions have been produced with the GPS High-precision Orbit determination Software Tools (GHOST, cf. Helleputte 2004) according to a procedure described by Montenbruck et al. (2005). The same software version and processing procedure is currently used at the German Space Operations Center (GSOC) of the Deutsches Zentrum für Luft- und Raumfahrt (DLR, Germany) to derive the TerraSAR-X orbit product (Yoon et al. 2009).

\subsection{Dynamic orbit determination}

The equation of motion of an Earth orbiting satellite including all perturbations reads in the inertial frame as

$\ddot{\mathbf{r}}=-G M \frac{\mathbf{r}}{r^{3}}+\mathbf{f}_{1}\left(t, \mathbf{r}, \dot{\mathbf{r}}, q_{1}, \ldots, q_{d}\right)$,

where $G M$ denotes the gravity parameter of the Earth, $\mathbf{r}$ and $\dot{\mathbf{r}}$ represent the satellite position and velocity, and $\mathbf{f}_{1}$ denotes the perturbing acceleration. The initial conditions $\mathbf{r}\left(t_{0}\right)=$ $\mathbf{r}\left(a, e, i, \Omega, \omega, T_{0} ; t_{0}\right)$ and $\dot{\mathbf{r}}\left(t_{0}\right)=\dot{\mathbf{r}}\left(a, e, i, \Omega, \omega, T_{0} ; t_{0}\right)$ at epoch $t_{0}$ are defined by six Keplerian osculating elements, e.g., $a, e, i, \Omega, \omega, T_{0}$. The parameters $q_{1}, \ldots, q_{d}$ in Eq. (1) denote additional dynamical orbit parameters considered as unknowns.

In the Bernese GPS Software, LEO POD is initiated by processing the spaceborne GPS code measurements of the LEO receiver to derive a first set of kinematic positions at the measurement epochs and to synchronize the LEO receiver clock with respect to GPS system time (Rockwell 1984). Based on a selected force model, the kinematic positions are approximated in the least-squares sense by numerically integrating the equation of motion using a high-order collocation method (Beutler 2005) and by adjusting the Keplerian osculating elements as well as the dynamical orbit parameters. The resulting a priori orbit $\mathbf{r}_{0}(t)$ is a particular solution of Eq. (1). Since only three constant empirical accelerations acting over the entire arc are adjusted in the radial, along-track, and cross-track directions as dynamical orbit parameters, the quality of the resulting a priori orbit is at the level of about $5 \mathrm{~m}$. No attempt is currently made in the Bernese GPS Software to take a priori models for atmospheric drag and radiation pressure into account to reduce the non-gravitational perturbations acting on the LEO satellites.

The final LEO orbit determination with the Bernese GPS Software is initiated by an orbit improvement process, i.e., the improved orbit $\mathbf{r}(t)$ is modeled as a truncated Taylor series with respect to the unknown orbit parameters $p_{i}$ about the a priori orbit $\mathbf{r}_{0}(t)$, which is represented by the parameter values $p_{i 0}$. The truncated Taylor series reads as

$\mathbf{r}(t)=\mathbf{r}_{0}(t)+\sum_{i=1}^{n} \frac{\partial \mathbf{r}_{0}(t)}{\partial p_{i}} \cdot\left(p_{i}-p_{i 0}\right)$,

where $n=6+d$ denotes the total number of unknown orbit parameters, i.e., the six Keplerian osculating elements and $d$ dynamical orbit parameters $q_{d}$. Efficient numerical integration techniques are applied to solve the variational equations (Beutler 2005) in order to obtain the partial derivatives in Eq. (2). These partials allow the eventual solution for corrections to the a priori orbit parameters $p_{i 0}$ (together with corrections for the non-orbit parameters) in a standard least-squares adjustment of GPS carrier phase observations only. Finally, the improved orbit may be obtained by either using Eq. (2) or by propagating the improved state vector by numerical integration and by taking into account the improved dynamical orbit parameters $q_{d}$. Note that the latter option yields an improved orbit which is again a particular solution of Eq. (1).

In the GHOST package a similar processing scheme is applied for LEO POD when using the standard batch leastsquares approach (cf. Kroes 2006). A major and important difference with respect to the Bernese GPS Software is the rigorous use of a priori models for the non-gravitational perturbations acting on the LEO satellites. Accelerations due to atmospheric drag and solar radiation pressure are computed using a simple "cannonball" model assuming uniform surface and mass properties. Atmospheric densities are computed with the Jacchia 71 model (Jacchia et al. 1971), whereas a conical Earth shadow model indicates whether solar radiation pressure has to be switched on. Scaling factors for atmospheric drag and solar radiation pressure are adjusted in the course of the LEO POD with the GHOST package, whereas constant empirical accelerations in the radial, along-track, and cross-track direction acting over the entire arc are determined by the Bernese GPS Software to compensate for the mean values of the unmodeled accelerations.

\subsection{Reduced-dynamic orbit modeling}

Purely dynamic LEO POD is currently not an option of the Bernese GPS Software due to the lack of models for 
atmospheric drag and radiation pressure, but also for the GHOST package the relevance is limited due to unavoidable deficiencies in the non-gravitational force models (Vallado and Finkelman 2008). Both software packages thus provide an implementation of the well-known reduced-dynamic approach (Wu et al. 1991) coping with the inexistent/deficient models. Reduced-dynamic orbit modeling techniques combine the strength of the GPS observations with the fact that satellite trajectories are particular solutions of a deterministic equation of motion.

Pseudo-stochastic orbit modeling techniques as described in detail by Jäggi et al. (2006) are used in the Bernese GPS Software to realize the LEO reduced-dynamic orbit determination. So-called pseudo-stochastic parameters, e.g., piecewise constant accelerations with a user-specified time resolution in the radial, along-track, and cross-track direction, are estimated together with the orbit and non-orbit parameters in the orbit improvement process and are added to the deterministic equation of motion for the eventual propagation of the improved state vector. In the GHOST package, a closely related procedure is applied. The piecewise constant accelerations are characterized in both software packages by a priori variances which constrain them to zero. It is important to note that the applied constraints are usually not very tight in the Bernese GPS Software due to the unmodeled non-gravitational accelerations. Thus, reduced-dynamic LEO orbits computed with the Bernese GPS Software are often regarded to be "more kinematic" than orbits computed with other software packages (e.g., Montenbruck et al. 2008).

\section{Antenna phase center modeling}

Modeling GPS observations requires the computation of the geometric distance between the antenna phase center location of the GPS satellite at signal emission time and the antenna phase center location of the receiving antenna at signal reception time (Teunissen and Kleusberg 1998). The phase center locations usually differ from the mechanical antenna reference points (ARPs), the difference vectors being conventionally described by a set of phase center corrections. Such a set of corrections consists of a phase center offset (PCO) vector $\mathbf{r}_{0}$ which defines the position of the "mean antenna phase center" with respect to the ARP and a consistent function $\phi$ which models the azimuth- and zenith/nadir-dependent phase center variations (PCVs). For convenience, both corrections are provided in an antenna-fixed coordinate system (see Sect. 3.1).

According to Rothacher et al. (1995), phase center corrections have some inherent degrees of freedom. For a receiver antenna, e.g., one set of corrections consisting of a $\mathrm{PCO}$ vector $\mathbf{r}_{0}$ and an azimuth- and zenith-dependent function $\phi(\alpha, z)$ may be transformed into a new set, consisting of $\mathbf{r}_{0}^{\prime}$ and $\phi^{\prime}(\alpha, z)$, that gives exactly the same results as the original set when applied to the GPS data:

$\mathbf{r}_{0}^{\prime}=\mathbf{r}_{0}+\Delta \mathbf{r}$

$\phi^{\prime}(\alpha, z)=\phi(\alpha, z)-\Delta \mathbf{r} \cdot \mathbf{e}+\Delta \phi$,

where $\Delta \phi$ and the offset vector $\Delta \mathbf{r}$ may be chosen arbitrarily and $\mathbf{e}$ denotes the unit vector pointing from the receiver to the satellite. The constant $\Delta \phi$ is an arbitrary phase offset for all directions that cannot be separated from the receiver clock, and $\Delta \mathbf{r}$ allows for an arbitrary location of the "mean" phase center in three coordinate directions. The constant $\Delta \phi$ is uniquely defined, e.g., by constraining $\phi(\alpha, z)$ in the boresight direction to zero, by constraining the sum over all azimuth and zenith angles to zero (zero-mean condition), or by any other convention.

\subsection{A priori modeling}

The a priori PCOs and PCVs for both the receiver and transmitter antennas are modeled in the respective antenna-fixed coordinate systems, which have their origin in the respective mechanical ARPs. The ARPs for TerraSAR-X (Montenbruck et al. 2009) and for the two GRACE satellites (W. Bertiger, private communication) are given in the respective satellitefixed coordinate systems. The latter differ slightly from those originally published by Bettadpur (2004).

The positive $z$-axis ("Up") of the right-handed antennafixed coordinate system coincides with the mechanical symmetry axis of the antenna and points along the boresight direction. The $y$-axis ("North") and $x$-axis ("East") point from the mechanical ARP into the respective directions, which depend on the specific mounting of the antennas. For the TerraSAR-X satellite the orientation of the GPS receiver antennas is documented by Montenbruck et al. (2009). For the GRACE satellites, however, the corresponding details of the antenna mounting are not traceable. The same alignment as for the TerraSAR-X main antenna has been assumed for both GRACE satellites in this study. For consistency with the adopted standards for terrestrial antenna corrections in a North-East-Up reference frame (cf. Schmid et al. 2005), the azimuth angle is counted in a clock-wise sense from the $y$-axis to the $x$-axis.

Satellite-specific GPS antenna offsets and nadir-dependent PCVs with a $1^{\circ}$ resolution (Schmid et al. 2007) are taken into account to model the phase center location of the GPS satellites. The LEO antenna phase center location is modeled according to the given PCOs and either with or without the $5^{\circ} \times 5^{\circ} \mathrm{PCV}$ s obtained from the ground calibration of the antenna/chokering combination used aboard TerraSAR-X (Montenbruck et al. 2009). In the Bernese GPS Software, the a priori phase patterns of both the receiver and transmitter antennas are represented as piecewise linear 
functions (polygon approach) with respect to the zenith/nadir and azimuth angles in the respective antenna-fixed coordinate systems. Note that the calibration of the GPS satellite antenna patterns is limited to nadir angles of $14^{\circ}$ as long as the calibration is based on terrestrial measurements. This is barely sufficient for the GRACE and TerraSAR-X GPS data analyzed in this study, where nadir angles of about $15^{\circ}$ are encountered at maximum. The antenna PCVs beyond $14^{\circ}$ have been held constant at their published values for $14^{\circ}$.

\subsection{Empirical modeling}

The PCO vectors, e.g., from the ground calibration, are introduced as fixed and only the PCVs are estimated. The physical meaning of the introduced vectors is given by the setup of the constraining condition for the PCVs. In this study, two different approaches, the so-called residual approach and the direct approach, are used for the in-flight determination of empirical corrections to the a priori PCVs of the LEO receiver antennas. The residual approach, well-known for LEO POD, has already been successfully applied, e.g., to improve JASON-1 orbits (Haines et al. 2004). Meanwhile, the Jet Propulsion Laboratory (JPL) applies this approach to the GRACE satellites, which serve as primary data source to derive PCVs for the GPS transmitter antennas (Haines et al. 2008). In this study, empirical PCVs for LEO receiver antennas are derived as bin-wise mean values from GPS carrier phase residuals, e.g., obtained from LEO reduced-dynamic POD. For long series of residuals, the bin size may be set as low as $1^{\circ}$.

The direct approach, not that much in use for LEO POD, has also already been applied to improve JASON-1 orbits (Luthcke et al. 2003). In this approach, corrections to the a priori PCVs of LEO receiver antennas are directly set up as estimation parameters when processing the GPS carrier phase measurements. In agreement with the a priori modeling described in Sect. 3.1, the empirical PCV corrections are modeled in this study as coefficients of piecewise linear functions in azimuth and elevation (polygon approach). As a long series of GPS carrier phase measurements is needed, daily normal equations have to be stored. The daily normal equations are then accumulated into a combined system covering the time span of interest, and may eventually be inverted to solve for the PCV corrections. Due to the fact that the storage requirements and the computational burden are significantly higher than for the residual approach, it was only feasible to derive PCV corrections with $2^{\circ}$ resolution $(8,280$ parameters), but not with $1^{\circ}$ resolution (32,760 parameters).

\section{GRACE simulation study}

A simulation study was conducted to assess the differences between the residual approach and the direct approach for the determination of LEO receiver antenna PCVs. For this purpose, the physical and mathematical models of the real data processing were used to simulate undifferenced GPS carrier phase observations for one GRACE satellite over one day on the $L_{1}$ and $L_{2}$ carrier frequencies down to an elevation cut-off angle of $0^{\circ}$. The GPS orbits from the final product line of the CODE analysis center (see Sect. 5) and a numerically integrated dynamic GRACE orbit served as the true GPS orbits and the true GRACE orbit to generate the simulated observations. For all scenarios the GPS observations were assumed to be error-free with PCOs and different sets of PCVs taken into account. Whereas the PCO values were assumed to be perfectly known for the subsequent GPS data analysis, the PCVs were neglected in the modeling of the carrier phase observations. The results of the residual approach are discussed in Sect. 4.1, those of the direct approach in Sect. 4.2.

\subsection{Residual approach}

\subsubsection{Simulation I}

Artificial PCVs with $5^{\circ}$ resolution and identical values on the carrier frequencies $L_{1}$ and $L_{2}$ have been imposed as true PCVs in a first simulation scenario. Figure 1 (left) shows that the chosen PCVs only depend on the azimuth angle $\alpha$ with constant values of $3 \mathrm{~mm}$ for $0^{\circ} \leq \alpha \leq 85^{\circ}$ and $-1 \mathrm{~mm}$ for $90^{\circ} \leq \alpha \leq 355^{\circ}$, respectively. According to Sect. 3.1, the polygon approach is applied to obtain the values for $85^{\circ}<$ $\alpha<90^{\circ}$ and $355^{\circ}<\alpha<360^{\circ}$. Although such a pattern cannot be found for real GPS receiver antennas, it is well suited to get insight into the performance of the residual approach. Note that an azimuth of $90^{\circ}$ points into the direction of flight.

In a first experiment, the true GRACE orbit is introduced as known to compute the $L_{3}$ carrier phase residuals as part of the standard least-squares adjustment process (see Sect. 2.1) with GPS carrier phase ambiguities and epoch-wise receiver clock corrections as the only unknowns. Figure 1 (right) shows the resulting $L_{3}$ carrier phase residuals (with opposite sign) when neglecting the artificial PCVs in the modeling of the carrier phase observations. Since no other mismodeling is present, Fig. 1 (right) directly reflects the impact of the unmodeled PCVs on the carrier phase residuals. We note that the resulting pattern is different from Fig. 1 (left), in particular for the bottom part of Fig. 1 (right) where the residuals show a trend from the left to the right-hand side.

Due to the neglect of the artificial PCVs in the carrier phase observation modeling, parts of them are absorbed by the epoch-wise estimated receiver clock corrections, which is reflected by a resulting clock drift, which in turn is responsible for the observed trend in the bottom part of Fig. 1 (right). In principle, the receiver clock offset should be constant in this simulation scenario, but whenever one of the tracked 
Fig. 1 Artificial PCVs of simulation I (left) and carrier phase residuals (right) in millimeters on the $L_{3}$ linear combination. Note the different color scales
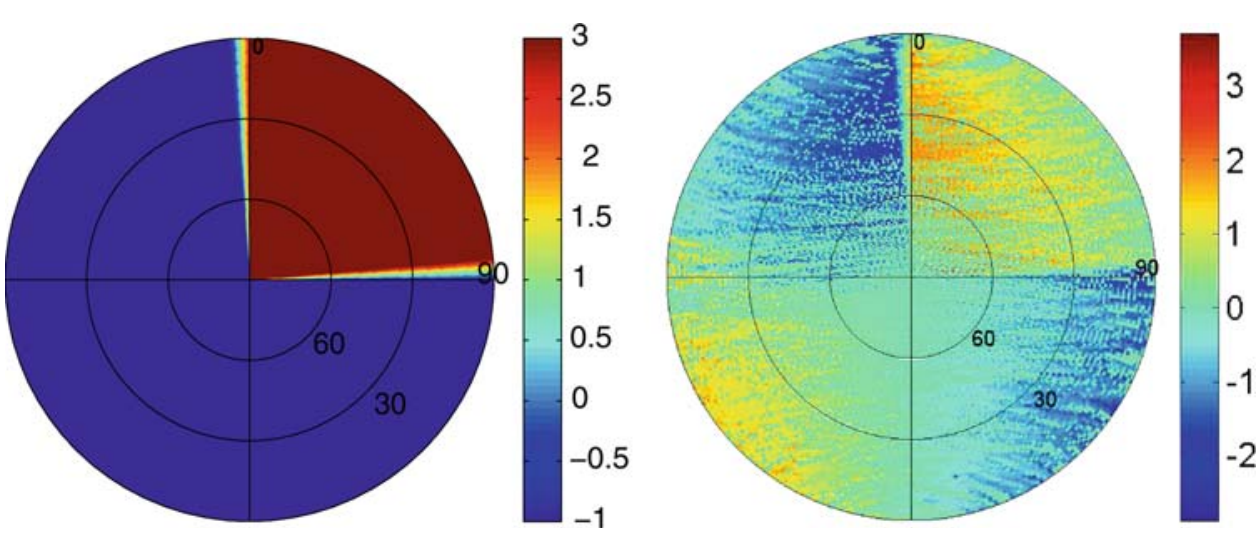
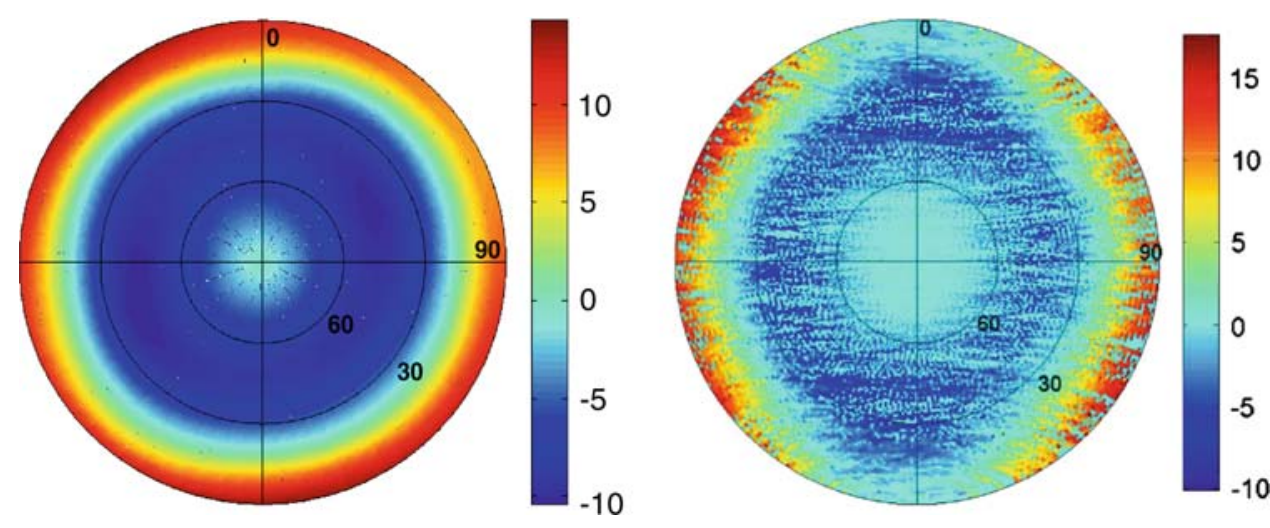

Fig. 2 Ground calibrated PCVs of simulation II (left) and carrier phase residuals (right) in millimeters on the $L_{3}$ linear combination
GPS satellites passes from the top right to the top left quadrant, a part of the linearly changing PCVs is absorbed by the receiver clock corrections. The magnitude of the induced clock change is given, in essence, by the number of simultaneously tracked GPS satellites. The total number of such passes per day determines the slope of the resulting clock drift.

\subsubsection{Simulation II}

PCVs on $L_{1}$ and $L_{2}$ with $5^{\circ}$ resolution stemming from the ground calibration of the antenna/chokering combination used aboard GRACE (see Sect. 3.1) have been used as true PCVs for a second simulation. Figure 2 (left) shows that the phase pattern of the $L_{3}$ linear combination is almost perfectly symmetric with respect to the boresight axis with values ranging from -10 to about $15 \mathrm{~mm}$.

As in Sect. 4.1.1, the true GRACE orbit is introduced as known to compute the $L_{3}$ carrier phase residuals when neglecting the PCVs from the ground calibration in the modeling of the carrier phase observations. Figure 2 (right) shows that significant parts of the neglected PCVs are mapped into the residuals, which preserve the structure of the original phase pattern quite well in this experiment. Pronounced deviations from Fig. 2 (left) are only observed in the top and bottom part of Fig. 2 (right), where the residuals are close to zero.
Due to the neglect of the ground calibrated PCVs in the carrier phase observation modeling, parts of them are absorbed by the carrier phase ambiguities. Assuming that carrier phase ambiguities are set up only once per GPS satellite pass, they absorb one mean value along each of the GPS satellite tracks. Since the orientation of the GRACE satellite is assumed to be perfectly aligned with the orbital frame in this simulation (an azimuth of $90^{\circ}$ pointing into the direction of flight), the GPS satellite tracks always proceed in a similar way from the right-hand to the left-hand side of Fig. 2 (right). Therefore, PCVs perpendicular to the flight direction are to some extent absorbed by the carrier phase ambiguities. In our experiment, no effect of the neglected PCVs can thus be seen in the top and bottom part of Fig. 2 (right).

The residuals were then used to generate an empirical correction map for the neglected PCVs with a resolution of $5^{\circ}$. Taking this first correction map into account, new residuals were subsequently computed to generate an incremental correction map with the same resolution to be added to the first correction map. This procedure was repeated several times. Figure 3 (left) shows the final PCV map after four iterations. The order of magnitude of the derived corrections as well as the shape of the pattern correspond quite well to the ground calibrated PCVs shown in Fig. 2 (left). The top and bottom part of Fig. 2 (left), however, could not be recovered with the residual approach. The largest part of the improvement was already achieved after the first iteration step. 
Fig. 3 Empirical PCVs in millimeters based on carrier phase residuals referring to the true GRACE orbit (left) and to a reduced-dynamic orbit (right), respectively (simulated data)
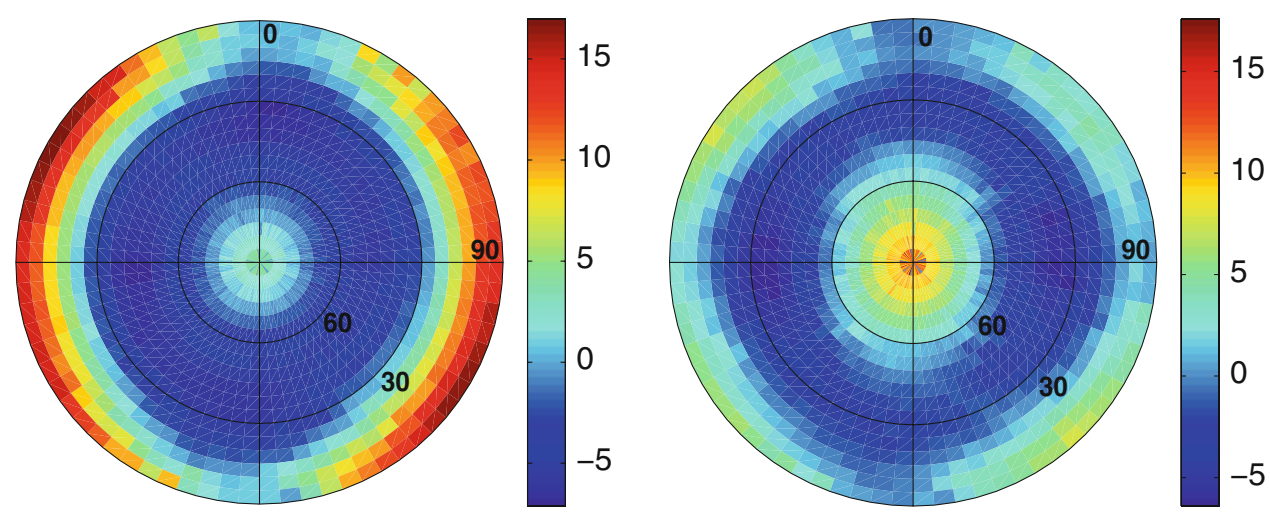

\subsubsection{Simulation III}

The same error-free GPS observations as in Sect. 4.1.2 were used to compute the GPS carrier phase residuals in a more realistic way when neglecting the PCVs from the ground calibration in the carrier phase observation modeling. Instead of introducing the true GRACE orbit as known, a reduceddynamic orbit was determined first to which the residuals refer. In analogy to Sect. 4.1.2, the residuals were then used to generate an empirical correction map of the neglected PCVs with a resolution of $5^{\circ}$. Taking this first correction map into account for a new reduced-dynamic orbit determination, new residuals were subsequently computed to generate an incremental correction map with the same resolution to be added to the first one. This procedure was repeated several times. As opposed to Sect. 4.1.2, several iteration steps are required in this experiment. The residuals of the reduced-dynamic POD are smaller than the residuals in Sect. 4.1.2, as significant parts of the neglected PCVs are also absorbed by the estimated orbit parameters. The more additional orbit parameters are solved for, the less the neglected PCVs are mapped into the carrier phase residuals. Instead, the orbit positions are affected via the estimated orbit parameters. Kinematic solutions, e.g., are particularly sensitive to a correct modeling of the antenna phase center location as no constraints are imposed by dynamic models on the epoch-wise estimated positions. In order to recover neglected PCVs with the residual approach, one has thus to strive for a most dynamic orbit representation with only few parameters, at least from the conceptual point of view. In reality, however, the force models (which are assumed to be perfectly known in this simulation) are not of a quality allowing for a pure dynamic POD. Therefore, reduced-dynamic orbits have to be used in order to avoid a severe and unnecessary degradation of the carrier phase residuals by additional orbit modeling errors.

Figure 3 (right) shows the final PCV map after four iterations. Whereas the order of magnitude of the derived corrections corresponds quite well to the ground calibration shown in Fig. 2 (left), the shape does not correspond to the correction

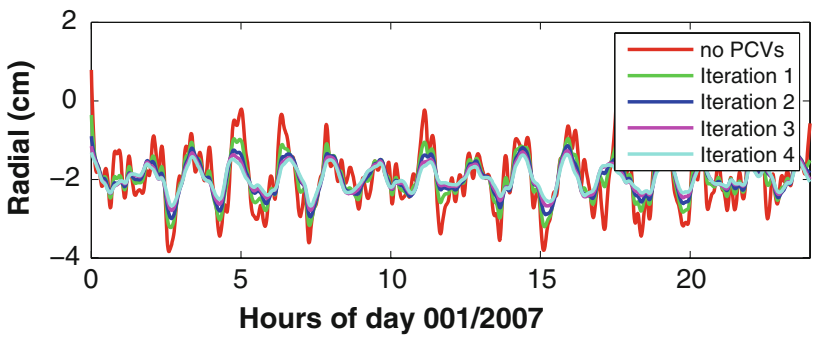

Fig. 4 Radial differences of the true orbit with respect to different reduced-dynamic orbits due to an insufficient modeling of PCVs (simulated data)

map obtained by introducing the true GRACE orbit as known (Fig. 3, left). This effect is explained by a PCO, which is induced by the neglected PCVs.

Figure 4 shows the radial differences between the true GRACE orbit and the different reduced-dynamic orbits used for the generation of the final PCV map shown in Fig. 3 (right). The curve in Fig. 4 with the largest magnitude corresponds to the solution which was computed without taking PCVs into account. The other curves, with decreasing magnitude, correspond to the solutions computed with the PCVs stemming from the first, second, third, and fourth iteration step, respectively. Pronounced orbit differences which are caused by the neglected PCVs, e.g., dominant once-per-revolution variations, are not only present in the radial direction, but also in the along-track and cross-track directions (not shown). The systematic offset of about $2 \mathrm{~cm}$ is, however, specific for the radial direction. Since the PCVs from the ground calibration were not constrained by a zero-mean condition but by a constraint in the boresight direction (see Sect. 3), a radial PCO is introduced in the modeling of the carrier phase observations when neglecting the PCVs, even if the antenna PCO vector is properly taken into account.

The fact that the induced PCO propagates one-to-one into a radial offset of the reduced-dynamic orbit solution can be explained by the LEO orbit parametrization used in the Bernese GPS Software, where constant accelerations acting over 
the entire orbital arc are estimated in the radial, along-track, and cross-track direction (see Sect. 2.1). As a consequence, the "datum" of the resulting trajectory is defined by the GPS measurements only, but not by the satellite dynamics. The radial offset of about $2 \mathrm{~cm}$ of the "higher-flying" reduceddynamic orbit in Fig. 4, e.g., is fully compensated by a constant radial acceleration of about $-70 \mathrm{~nm} / \mathrm{s}^{2}$. Therefore, one is not sensitive to erroneous PCOs when using the current orbit parametrization of the Bernese GPS Software for LEO POD.

Figure 4 also shows that the orbit differences gradually decrease when taking the empirical PCVs from the different iteration steps into account. The most pronounced improvements are encountered in the first iteration step, but each additional iteration step further reduces the orbit differences. However, for the reasons mentioned, the radial offset cannot be reduced due to the orbit parametrization.

\subsection{Direct approach}

\subsubsection{Simulation I and II}

The scenarios of the simulations I and II were used to directly estimate PCV parameters for the LEO antenna. The true GRACE orbit was introduced as known, and PCVs were estimated on a grid with a resolution of $5^{\circ}$. In order to prevent the system of normal equations from becoming singular, the sum over all azimuth and zenith angles was constrained to zero (zero-mean condition). This is necessary, as the constant $\Delta \phi$ in Eq. (3) is arbitrary.

For both simulations the neglected PCVs are correctly recovered by the direct approach. The directly estimated PCVs agree with the artificial PCVs for the scenario of simulation I. For the scenario of simulation II the recovered PCVs differ by a constant offset of about $2.7 \mathrm{~mm}$ from the true PCVs from the ground calibration. The result from simulation II is, so to speak, the normal case, because PCVs need not necessarily meet the applied zero-mean condition. Due to the arbitrary constant $\Delta \phi$ in Eq. (3), this offset is irrelevant and fully absorbed by the estimated receiver clock correction. For both simulations the associated carrier phase residuals are on a level of about $10^{-8} \mathrm{~m}$, rendering additional iteration steps obsolete. As opposed to the residual approach, the direct PCV estimation is not harmed by the estimation of the GPS carrier phase ambiguities and the epoch-wise receiver clock corrections. Thus, from the conceptual point of view, the direct approach is superior to the residual approach.

\subsubsection{Simulation III}

The scenario of simulation III was used to directly estimate PCV parameters under more realistic conditions. Instead of introducing the true GRACE orbit as known, the degraded trajectory from the reduced-dynamic POD ignoring the PCVs for the LEO antenna was introduced as known, and PCVs were estimated on a grid with a resolution of $5^{\circ}$ by applying a zero-mean condition. Although this condition is sufficient from the theoretical point of view, it makes sense to prevent weakly determined PCV parameters, e.g., related to bins near the elevation cut-off angle with small numbers of GPS observations, from shifting all other PCV parameters by unrealistic values due to the zero-mean condition. Therefore, an additional a priori standard deviation (STD) of $1 \mathrm{~cm}$ is imposed to weakly constrain all PCV parameters to zero.

The directly estimated PCVs are similar to the PCVs from the first iteration step of the residual approach. Taking them into account for a subsequent reduced-dynamic orbit determination confirms that the differences between the newly computed reduced-dynamic orbit and the true orbit are slightly smaller but comparable to those shown in Fig. 4. In analogy to Sect. 4.1.3 several iteration steps are required, based on the reduced-dynamic orbits and the previously estimated PCVs, to further improve the quality of the PCV estimates.

Figure 5 shows the final map after four iteration steps. In order to enable a better comparison with the results from Sect. 4.1.3, the PCVs are shown as block-wise constant values instead of piecewise linear values. Compared to Fig. 3 (right) the PCVs show a very homogeneous structure with an almost perfect rotational symmetry. The top and bottom parts of Fig. 5 are no longer degraded due to the simultaneous estimation of PCV parameters and carrier phase ambiguities. However, the shape of the PCVs is again dominated by the unavoidable PCO (cf. Sect. 4.1.3). A comparison of the underlying reduced-dynamic orbits of all individual iteration steps (not shown) with those from Sect. 4.1.3 reveals a slightly better quality of the individual orbits for the first few iteration steps. Although not dramatic in terms of the

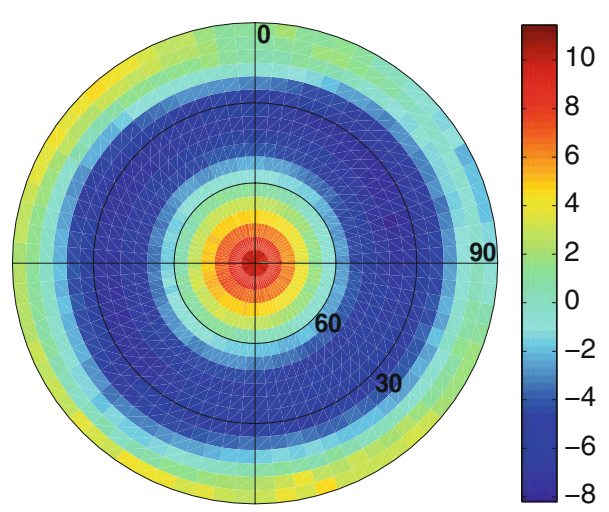

Fig. 5 Empirical PCVs in millimeters based on the direct approach with fixed reduced-dynamic orbits (simulated data) 
orbit quality, the direct approach is superior to the residual approach in some respects.

\subsubsection{Outlook}

The approach performed in Sect. 4.2.2 is still limited, as the LEO positions were kept fixed on a (degraded) trajectory computed before-hand with no or only imperfect PCV information taken into account. Although the impact of the erroneous positions can be reduced in subsequent iteration steps, it cannot be eliminated completely. Only a simultaneous estimation of PCV, orbit and clock parameters as well as carrier phase ambiguities has the potential to circumvent this problem. Due to the inherent degrees of freedom of PCV parameters (cf. Sect. 3), this is not possible without putting additional constraints on selected orbit or PCV parameters. A radial shift $\Delta \mathbf{r}=(0,0, \Delta h)$, e.g., can be absorbed by the estimated PCVs and the constant radial acceleration acting over the entire orbital arc, whereas a shift in the along-track direction can be absorbed by the estimated PCV parameters and the argument of latitude, one of the Keplerian osculating elements. Although such a simultaneous estimation is feasible from the practical point of view, the "datum" of the resulting orbit cannot be properly realized when using the current LEO orbit parametrization of the Bernese GPS Software. A more dynamic modeling would be a prerequisite to avoid the set-up of constant accelerations acting over the entire arc, so that some existing degrees of freedom could be removed and, thus, a simultaneous estimation could be enabled.

\section{LEO POD based on zero-difference observations}

The final GPS orbits and the $30 \mathrm{~s}$ high-rate satellite clock corrections from the CODE analysis center (Dach et al. 2009) were used together with the GRACE gravity field model EIGEN-CG03C (Förste et al. 2005) and attitude data from the
GRACE star trackers to process undifferenced GRACE GPS carrier phase tracking data with 30 s sampling covering the year 2007. An elevation cut-off angle of $5^{\circ}$ and a threshold of 5 for the signal-to-noise ratio was applied to select GPS data of high quality. TerraSAR-X data of the main antenna were made available for the days 270-280 of the year 2007 by GSOC and processed in analogy to the GRACE data. A development version of the Bernese GPS Software (Dach et al. 2007) was used to estimate the orbit parameters mentioned in Sect. 2 together with all other relevant parameters like receiver clock corrections and real-valued carrier phase ambiguities. The pseudo-stochastic parameters were set up as piecewise constant accelerations in the radial, along-track, and cross-track directions with a time resolution of $6 \mathrm{~min}$ and an a priori STD of $5 \mathrm{~nm} / \mathrm{s}^{2}$ (see Sect. 2.2)

\subsection{Internal validation}

Let us first study the impact of the a priori antenna phase center modeling on reduced-dynamic and kinematic orbit solutions computed with the Bernese GPS Software. Internal validation criteria are analyzed for three different solution types computed from GRACE A, GRACE B, and TerraSAR-X GPS data covering a time period of 21 and 11 days, respectively (see Table 1). For the first solution type, denoted by "ARP", PCO and PCV values from the ground calibration are neglected, implying that the antenna phase center location coincides with the antenna reference point. For the second solution type, denoted by "+PCO ground calib.", the PCO values from the ground calibration are taken into account. And for the third solution type, denoted by "+PCV ground calib.", the PCV values from the ground calibration are also considered.

The internal validation criteria are the root mean square (RMS) errors of the $L_{3}$ GPS carrier phase residuals obtained from the kinematic and the reduced-dynamic orbit determination as well as the differences between the kinematic and
Table 1 Carrier phase residuals and difference between kinematic (KIN) and reduced-dynamic $(\mathrm{RD})$ orbit solutions [days 090-110, 2007 for GRACE and days 270-280, 2007 for TerraSAR-X (TSX)]

\begin{tabular}{llllll}
\hline Spacecraft & $\begin{array}{l}\text { Phase center } \\
\text { model }\end{array}$ & $\begin{array}{l}\text { Residuals } \\
\text { KIN RMS } \\
(\mathrm{mm})\end{array}$ & $\begin{array}{l}\text { Residuals } \\
\text { RD RMS } \\
(\mathrm{mm})\end{array}$ & $\begin{array}{l}\text { KIN-RD } \\
\text { RMS } \\
(\mathrm{cm})\end{array}$ & $\begin{array}{l}\text { KIN-RD } \\
\text { cross-track } \\
\text { mean }(\mathrm{cm})\end{array}$ \\
\hline GRACE A & ARP & 3.12 & 4.50 & 2.21 & 1.40 \\
& +PCO ground calib. & 3.12 & 4.50 & 2.21 & 1.40 \\
& +PCV ground calib. & 3.59 & 6.53 & 2.39 & 1.34 \\
GRACE B & ARP & 2.43 & 3.70 & 3.08 & 1.39 \\
& +PCO ground calib. & 2.43 & 3.69 & 3.08 & 1.38 \\
& +PCV ground calib. & 2.83 & 4.96 & 3.47 & 1.16 \\
& ARP & 4.98 & 6.73 & 3.29 & 1.61 \\
& + PCO ground calib. & 4.98 & 6.73 & 3.29 & 1.61 \\
& + PCV ground calib. & 4.68 & 6.32 & 2.72 & 1.38 \\
\hline
\end{tabular}


the reduced-dynamic orbit positions in terms of RMS error and mean offset in cross-track direction.

Table 1 shows that there is no difference between the first and the second solution type for all LEOs (apart from rounding effects). Reduced-dynamic and kinematic orbit differences between the two solution types would reveal that both the kinematic and the reduced-dynamic orbits are shifted in space by the PCO vector. Whereas such an effect has to be expected for a kinematic solution, which is linked to the GPS constellation by the GPS measurements only, in the reduceddynamic case it is caused by estimating the three constant accelerations in the radial, along-track, and cross-track directions (as explained in Sect. 4.1.3). Therefore, also in the case of a reduced-dynamic solution the internal validation is the same for the two solution types when using the Bernese GPS Software. Note that the results obtained by other software packages may show a different behavior when relying on a more dynamic modeling as illustrated by Montenbruck et al. (2009) for the same time period with GPS data from TerraSAR-X.

As a consequence of the fact that the "datum" of a reduceddynamic solution is only defined by the GPS measurements, as it is also the case for a kinematic solution, no offsets between reduced-dynamic and kinematic solutions are present in the radial and in the along-track direction. The last column of Table 1 shows, however, that offsets exist in the cross-track direction. Despite the estimation of a constant cross-track acceleration there is a discrepancy between the "cross-track datum" of a reduced-dynamic and a kinematic solution. This effect is further commented in Sect. 5.2.3.

Furthermore, Table 1 illustrates some basic properties of kinematic and reduced-dynamic solutions. Due to the epochwise estimation of the kinematic positions, resulting in a very large number of estimated parameters, the degree of freedom in the least-squares adjustment is considerably smaller than for a reduced-dynamic orbit determination. Due to the smaller redundancy the RMS error of the carrier phase residuals from a kinematic orbit determination is smaller than from a reduced-dynamic one. As mentioned in Sect. 4.1.3, GPS carrier phase measurement errors are thus rather absorbed by the large number of estimated positions than reflected in the carrier phase residuals.

Table 1 finally shows that the application of the PCVs from the ground calibration improves the internal validation for TerraSAR-X, but not for the two GRACE satellites. Although all satellites are equipped with the same antenna/chokering combination, the actual PCVs obviously also depend on the antenna/chokering integration into the satellite environment. In the case of TerraSAR-X the chokering is mounted on-top of the satellite surface, but for GRACE it is lowered with respect to the satellite surface.

\subsection{Empirical PCVs obtained with the residual approach}

Let us now study the impact of PCVs which are empirically derived in-flight from GPS carrier phase residuals computed with the Bernese GPS Software. For this purpose, two different types of reduced-dynamic solutions covering a time period of 1 year are computed for the two GRACE satellites. For the first solution type, starting with solution "NO", the PCO values from the ground calibration are applied without taking the PCV information from the same calibration into account. Subsequent solutions, denoted by "N1" to "N4", take the empirical PCVs into account, which were iteratively derived from the residuals stemming from the previous reduced-dynamic solution according to Sect. 4.1.3.

For the second solution type (identifier "W"), the same procedure is applied as for the solutions of type "N", but with the PCV information from the ground calibration additionally taken into account (as a priori information for the computation of the solution "W0"). For all solution types the empirical PCVs are derived with resolutions of both $5^{\circ}$ and $1^{\circ}$.

Figure 6 shows the empirical PCVs derived after four iterations from the reduced-dynamic solutions " $N$ " for GRACE A and GRACE B, respectively. With the exception of 3 days, which had to be excluded, e.g., due to maneuvers, the
Fig. 6 Empirical PCVs in millimeters for the trailing satellite GRACE A $(l e f t)$ and the leading satellite GRACE B (right) based on 362 days of $L_{3}$ carrier phase residuals in the year $2007\left(1^{\circ} \times 1^{\circ}\right.$ resolution, residual approach)
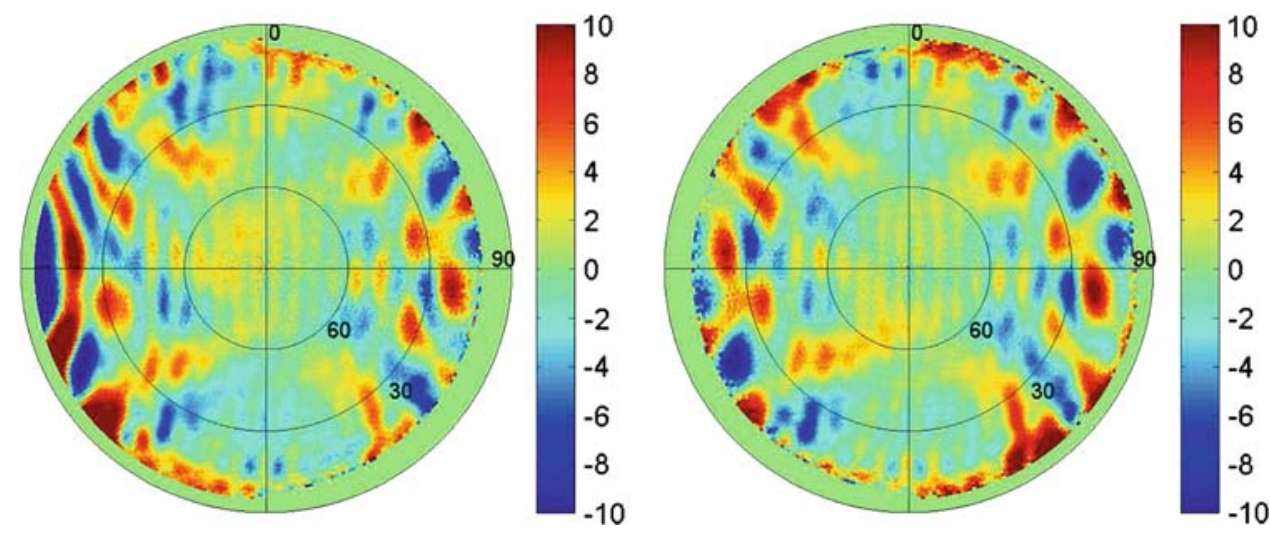
residuals of the entire year 2007 contributed to the generation of the $1^{\circ} \times 1^{\circ}$ correction maps as the repeatability of the observed patterns in the antenna-fixed coordinate system was found to be very high. Note that an azimuth of $90^{\circ}$ and $270^{\circ}$ points into the direction of flight for GRACE A and GRACE B, respectively, which explains the different location of the $10^{\circ}$ elevation cut-off angle for raising GPS satellites given by the settings of the GRACE receiver firmware.

The patterns of the two GRACE antennas shown in Fig. 6 are similar except for the left part of Fig. 6 (left) pointing to the active occultation antenna of GRACE A. Due to receiver internal cross-talk, measurements of BlackJack/ IGOR receivers are known to be affected by systematic, multipath-like errors during the operation of occultation antennas (Montenbruck and Kroes 2003). Apart from that, the structures in the patterns of GRACE A and B are almost identical. As the two GRACE satellites are identical in construction and use the same type of GPS antenna and receiver, one would expect that. Surprisingly, however, the pattern is not symmetric with respect to the body axes as should be expected from the design of the GRACE satellites. The reason for the patchy structure is most probably related to the GRACE receiver antennas but not to the transmitter antennas of the GPS satellites since azimuth angles in the transmitter and receiver antenna systems are decorrelated for extended GRACE data sets. The patterns obtained from data of the entire year 2007 were found to be fairly constant in time, which agrees with earlier studies based on data of the year 2003 (Jäggi et al. 2007). Also, the patterns are similar to those obtained from the earlier data, although, at that time, they had to be computed with the relative phase center model for GPS antennas and by neglecting PCVs for the transmitter antennas. Multipath is thus a plausible source for the observed systematic errors. This conclusion is also supported by Haines et al. (2008), who found patterns of carrier phase $L_{3}$ residuals that resemble the results from multipath simulations. The reason for the overlaid vertical stripes is not known to us.

\subsubsection{Orbit validation with SLR data}

Independent SLR measurements of the entire year 2007 are used to compare the computed ranges between the GRACE satellites and the SLR ground stations with the observed ranges. Due to obvious differences in the performance of the available SLR stations of the tracking network of the International Laser Ranging Service (ILRS, Pearlman et al. 2002), a subset of high-quality stations is selected to provide a realistic measure of the orbit errors. Based on solution "N0" of GRACE A it was found that the majority of the SLR stations scored overall SLR RMS errors between 1.3 and $3 \mathrm{~cm}$. These stations (among them well-known sites like Herstmonceaux, Graz, Greenbelt, Mount Stromlo, Yarragadee,
Table 2 SLR residuals (RMS and mean) of reduced-dynamic orbit solutions (identifier "N") for days 001-365, 2007

\begin{tabular}{llll}
\hline Spacecraft & Solution ID & $\begin{array}{l}\text { SLR residuals } \\
\text { RMS }(\mathrm{cm})\end{array}$ & $\begin{array}{l}\text { SLR residuals } \\
\text { mean }(\mathrm{cm})\end{array}$ \\
\hline GRACE A & N0 & 1.81 & 0.65 \\
& N1 $\left(5^{\circ} \times 5^{\circ}\right)$ & 1.81 & 0.65 \\
& N4 $\left(5^{\circ} \times 5^{\circ}\right)$ & 1.85 & 0.67 \\
GRACE B & N0 & 2.02 & 0.85 \\
& N1 $\left(5^{\circ} \times 5^{\circ}\right)$ & 1.99 & 0.85 \\
& N4 $\left(5^{\circ} \times 5^{\circ}\right)$ & 1.95 & 0.83 \\
\hline
\end{tabular}

Table 3 SLR residuals (RMS and mean) of reduced-dynamic orbit solutions (identifier "W") for days 001-365, 2007

\begin{tabular}{llll}
\hline Spacecraft & Solution ID & $\begin{array}{l}\text { SLR residuals } \\
\text { RMS }(\mathrm{cm})\end{array}$ & $\begin{array}{l}\text { SLR residuals } \\
\text { mean }(\mathrm{cm})\end{array}$ \\
\hline GRACE A & W0 $\left(5^{\circ} \times 5^{\circ}\right)$ & 1.77 & -0.14 \\
& W1 $\left(5^{\circ} \times 5^{\circ}\right)$ & 1.68 & -0.15 \\
GRACE B & W0 $\left(5^{\circ} \times 5^{\circ}\right)$ & 1.87 & 0.09 \\
& W1 $\left(5^{\circ} \times 5^{\circ}\right)$ & 1.79 & 0.10 \\
\hline
\end{tabular}

and Zimmerwald) contribute about $77 \%$ of the available observations and were thus used for the validation of the orbits.

Table 2 shows that the results of the SLR validation are almost identical for the different solutions of type "N". The mean SLR RMS errors of about 1.8 and $2 \mathrm{~cm}$ indicate a high quality of the GRACE orbits even if PCVs are completely neglected in the carrier phase observation modeling. The density and accuracy of the SLR measurements is, however, apparently insufficient to reveal the differences caused by the application of empirical PCVs. Expressed in a positive manner, no degradation can be detected by the SLR validation when applying the empirical PCVs. Table 2 also confirms that systematic offsets of about 0.65 and $0.85 \mathrm{~cm}$ remain in the SLR residuals, if only the PCOs from the ground calibration are taken into account but not the PCVs from that calibration. It is important to note that the empirically derived PCVs with the Bernese GPS Software cannot correct for these offsets as shown by the solutions "N1" and "N4".

Table 3 shows that the results of the SLR validation are similar for the solutions of type "W". However, in contrast to Table 2 the solutions of type "W" are almost free of systematic offsets in the SLR residuals. This fact underlines that the complete set of PCO and PCV values from the ground calibration has to be applied (if available). Empirically derived PCVs from POD solutions may then be applied in addition to further improve the orbit quality, which is indicated in Table 3 by a very small decrease of the scatter of the SLR residuals for the solution "W1". No further iterations have been performed for the solutions of type "W". 


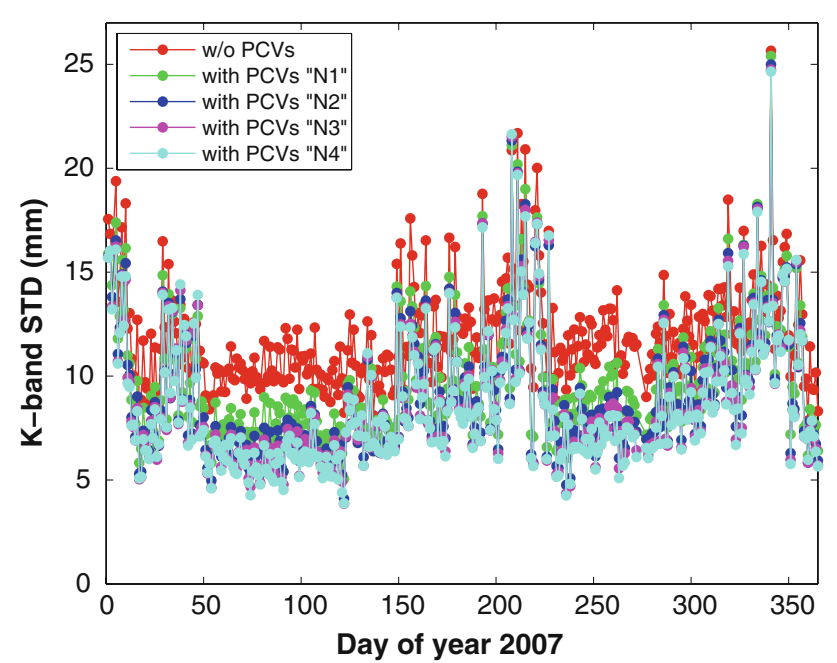

Fig. 7 Daily K-band range STD for distances between the zero-difference reduced-dynamic GRACE A and B orbits (solutions "NO" to "N4")

\subsubsection{Orbit validation with $K$-band data}

Independent K-band measurements are used to compare the orbit-derived distances between the two GRACE satellites with the biased ranges which are directly observed by the Kband ranging system. Figure 7 shows the daily K-band range STD obtained from distances computed every $5 \mathrm{~s}$ between the reduced-dynamic GRACE A and B orbit positions for the solutions of type "N" with a PCV resolution of $1^{\circ}$. Apart from 7 days that were excluded, e.g., due to maneuvers or problematic K-band data (Flechtner et al. 2007), the data of the entire year 2007 were used for the K-band validation. The mean K-band range STDs for the solutions "N0" to "N4" are 11.9, 9.7, 9.0, 8.7, and $8.6 \mathrm{~mm}$, respectively. Since the patchy structure in the empirically determined GRACE PCVs has angular extensions which are considerably larger than $1^{\circ}$ (cf. Fig. 6 ), there is almost no degradation $(\approx 0.1 \mathrm{~mm}$ $\mathrm{STD}$ ) in the K-band validation results when using PCVs with a resolution of $5^{\circ}$ instead of $1^{\circ}$. In essence the same significant reduction of the $\mathrm{K}$-band residuals can be achieved in both cases. The reduction is most obvious for periods when the data quality is excellent, e.g., for days 60-120 where the mean K-band range STD drops from 10.3 to 7.6, 6.6, 6.2, and $5.9 \mathrm{~mm}$, respectively. For such periods the improvement between the solutions "N0" and "N4" is almost a factor of 1.7. Note that almost the same K-band validation results are obtained (differences of $\approx 0.1 \mathrm{~mm}$ in the K-band range STD, not shown) when using the PCVs from the ground calibration as a priori values (solutions of type "W").

\subsubsection{Issues related to the cross-track direction}

The quality of orbits in the cross-track direction is difficult to assess as independent measurements, such as K-band or

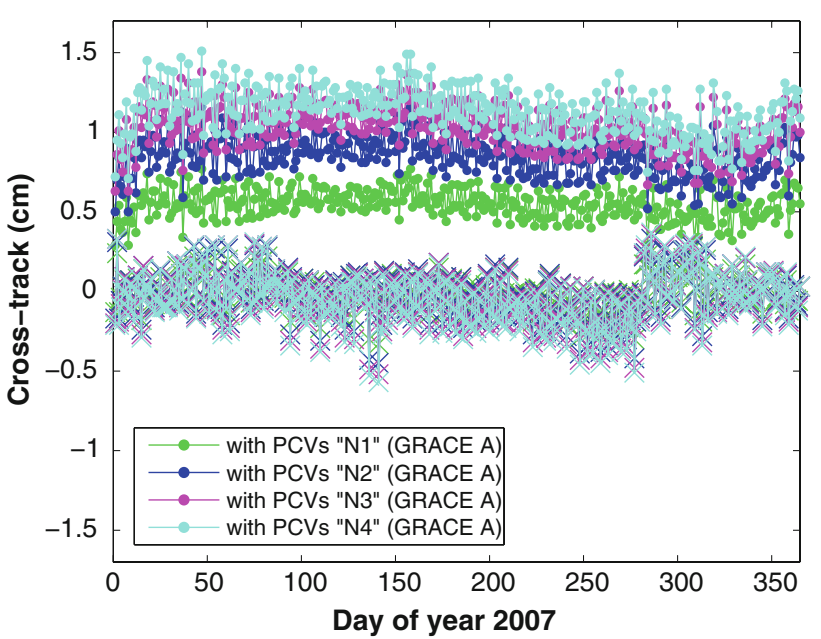

Fig. 8 Daily mean cross-track shifts (solutions "N1" to "N4") with respect to the reduced-dynamic solution "N0" for GRACE A (filled circles) and GRACE B (crosses)

SLR data, are hardly sensitive in this direction. Cross-track orbit errors are thus very likely to remain completely undetected. The comparison between reduced-dynamic and kinematic orbits in Table 1 indicated, however, that rather large systematic cross-track shifts may occur. Experiments with both the Bernese GPS Software and the GHOST package confirmed that the cross-track component is very sensitive to the POD settings in the presence of unmodeled systematic errors, which may also result in cross-track shifts between reduced-dynamic and kinematic orbit solutions as observed in Sect. 5.1.

Let us now illustrate the impact of the empirically determined PCVs on the "datum" of the orbit solutions. As no systematic shifts were found for the radial and the alongtrack direction, Fig. 8 concentrates on the daily mean crosstrack shifts between the reduced-dynamic solution "NO" and the solutions "N1" to "N4". Whereas all the solutions for GRACE B more or less agree with each other, the GRACE A solutions "N1" to "N4" are systematically shifted with respect to the solution "N0" by $5.5,8.5,10.3$, and $11.3 \mathrm{~mm}$, respectively.

Although not obvious from Fig. 6, the empirical PCVs for GRACE A seem to be able to change the cross-track "datum" of the GRACE A orbit solutions, which is an indication for the presence of unmodeled systematic errors in the GPS data. We believe that small-scale variations (smaller than about $30^{\circ}$ ) are rather well captured by PCVs derived from GPS carrier phase residuals, but large-scale variations might erroneously or not at all be captured by this approach.

\subsection{Empirical PCVs obtained with the direct approach}

Let us now study the impact of PCVs which are empirically estimated in-flight from GPS carrier phase measurements 
Fig. 9 Empirical PCVs in millimeters for the trailing satellite GRACE A (left) and the leading satellite GRACE B (right) based on 362 days of $L_{3}$ carrier phase measurements in the year $2007\left(5^{\circ} \times 5^{\circ}\right.$ resolution, direct approach). Note the different color scales

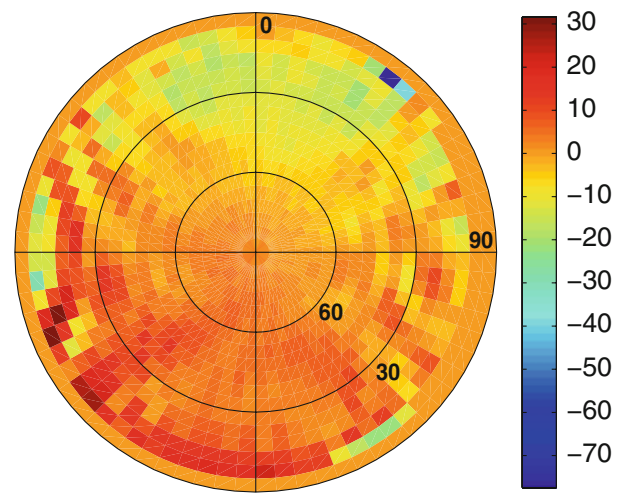

with the Bernese GPS Software. For this purpose, reduceddynamic solutions covering a time period of 1 year are computed for the two GRACE satellites. Starting from solution type "N0", subsequent solutions, denoted by "D1" to "D4", take the empirical PCVs into account, which are iteratively estimated from the GPS carrier phase measurements by introducing the trajectory from the previous reduced-dynamic orbit determination as known according to Sect. 4.2.2. The empirical PCVs are derived with a resolution of only $5^{\circ}$ to save computation time.

Figure 9 shows the empirical PCVs derived within four iterations from the different reduced-dynamic solutions " $D$ " for GRACE A and B, respectively. A comparison with the analogous PCVs derived from GPS carrier phase residuals $\left(5^{\circ}\right.$ resolution, not shown) reveals similar small-scale variations, as could be expected from the results of the simulation study (see Sect. 4.2.2). In contrast to the residual approach, however, the patterns obtained with the direct approach are governed by large systematic effects, e.g., a significant trend which can be recognized from the top to the bottom part of Fig. 9 (left). We believe that those effects reflect the unmodeled systematic errors already mentioned in Sect. 5.2.3. The directly estimated PCV parameters absorb them and, as opposed to the residual approach, prevent the orbits from being shifted in the cross-track direction. The solutions "D1" to "D4" show only a small and almost constant mean crosstrack offset with respect to the solution "N0" of $2.4 \mathrm{~mm}$ for GRACE A and $1.6 \mathrm{~mm}$ for GRACE B, respectively. Unfortunately, it is difficult to interpret the directly estimated PCVs in the presence of the overlaid large systematic errors. Their origin is not clear so far.

\subsubsection{Orbit validation with $K$-band data}

Independent K-band measurements are used in analogy to Sect. 5.2.2 to compare the orbit-derived distances between the two GRACE satellites with the observed biased ranges. In essence the same significant reduction of the K-band residuals is achieved after four iterations, but the convergence is achieved more rapidly as predicted by the simulation study (see Sect. 4.2.2). The mean K-band range STDs for the solutions "D1" to "D4" are 9.2, 8.7, 8.6, and $8.6 \mathrm{~mm}$, respectively. Also for the excellent data period from day 60 to 120 (see Fig. 7) a small benefit can be detected for the first iteration steps, where the K-band range STD drops from 10.3 to 6.9, $6.2,6.0$, and $5.9 \mathrm{~mm}$, respectively. After several iterations, however, the residual approach and the direct approach both give the same K-band validation results.

\section{GRACE baseline determination}

The GPS final orbits from the CODE analysis center are used together with the GRACE gravity field model EIGENCG03C and attitude data from the GRACE star trackers to process doubly differenced GRACE GPS carrier phase tracking data covering the time period of the year 2007. Reduceddynamic GRACE baseline solutions are determined with the Bernese GPS Software according to a procedure described by Jäggi et al. (2007). The positions of one GRACE satellite are kept fixed on a zero-difference reduced-dynamic solution from Sect. 5.2, which allows the estimation of reduceddynamic orbit parameters for the other satellite together with all other relevant parameters from the doubly differenced GRACE observations only. The carrier phase ambiguities are thereby resolved to their integer values. This option is realized by analyzing the Melbourne-Wübbena linear combination to first resolve the wide-lane ambiguities, which are subsequently introduced as known to resolve the narrow-lane ambiguities simultaneously to the reduceddynamic orbit determination. For optimal results, the differences between the piecewise constant accelerations of GRACE A and B are tightly constrained to $1 \mathrm{~nm} / \mathrm{s}^{2}$.

Two different reduced-dynamic baseline solutions with resolved carrier phase ambiguities are computed for the time period of the year 2007. For the first solution, denoted in analogy to Sect. 5.2 by "N0", only PCO values from the ground calibration are applied. The positions of GRACE A are kept fixed on the zero-difference solution "NO". For the second solution, denoted by "N4", the empirical PCVs derived 
Fig. 10 Empirical differential PCVs $(l e f t)$ in millimeters between the two GRACE satellites $\left(2^{\circ} \times 2^{\circ}\right.$ resolution, direct approach) and daily K-band range STD (right) for distances between the double-difference reduced-dynamic GRACE A and B orbits (solutions "NO" and "N4")

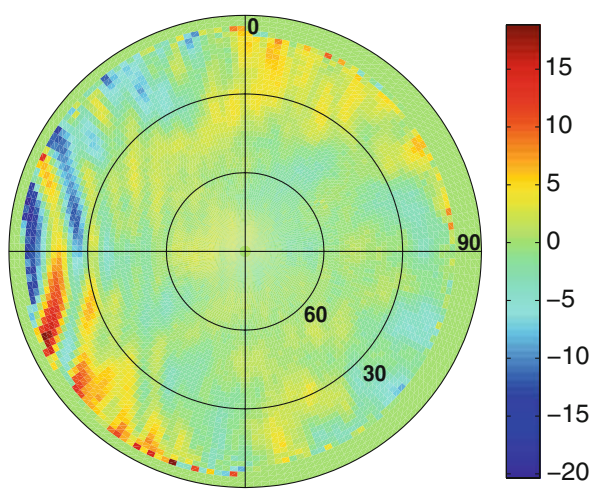

in-flight within four iterations from the different zerodifference solutions " $N$ " (cf. Sect. 5.2) are taken into account. The positions of GRACE A are kept fixed on the zero-difference solution "N4".

Note that it is also possible to directly estimate differential PCVs from doubly differenced GPS carrier phase measurements with the Bernese GPS Software, e.g., by introducing the reduced-dynamic baseline solution "N0" with resolved carrier phase ambiguities as known. Figure 10 (left) illustrates that in such a case the directly estimated differential PCVs, shown in the antenna-fixed coordinate system of GRACE A with a resolution of $2^{\circ}$, are not affected by obvious systematic errors as observed in the zero-difference processing, provided that baseline vectors with resolved carrier phase ambiguities are used for the PCV estimation. Whereas the impact of the active occultation antenna of GRACE A is again reflected in the left-hand side of Fig. 10 (left), the right-hand side reveals the small differences between the antenna patterns of the two GRACE satellites.

\subsection{Baseline validation with K-band data}

The K-band ranging system is used to compare the reduceddynamic baseline length with the ultra-precise K-band range measurements. Figure 10 (right) shows the daily K-band range STDs obtained from distances computed every $5 \mathrm{~s}$ for the baseline solutions "N0" and "N4". Apart from 8 days excluded due to difficulties with the ambiguity resolution, the solutions of the entire year 2007 were used for the validation. The mean K-band range STDs for the solutions "NO" and "N4" are 1.10 and $0.81 \mathrm{~mm}$, respectively. For periods when the data quality is excellent, e.g., for the period between the days 70 and 100, the mean K-band range STDs are even as small as 0.90 and $0.61 \mathrm{~mm}$, respectively. The value of $0.90 \mathrm{~mm}$ obtained without empirical PCVs taken into account is in good agreement with the $0.88 \mathrm{~mm}$ found by Jäggi et al. (2007) for a period of 55 days of data with excellent quality in the year 2003. Figure 10 (right) shows that it is feasible, however, to further improve the reduced-dynamic baseline

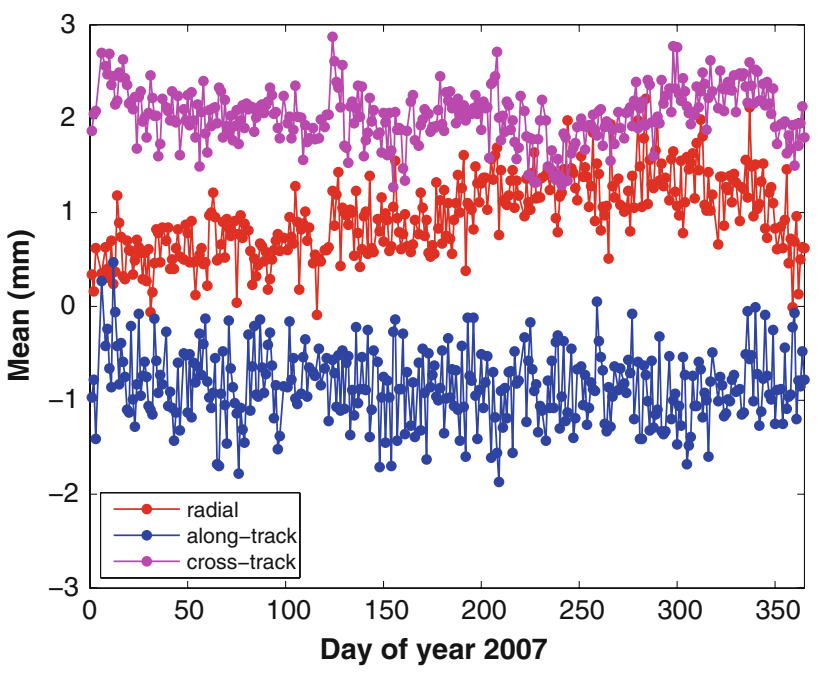

Fig. 11 Daily means of differences between AIUB and GSOC baseline vectors based on resolved carrier phase ambiguities

estimates by taking the empirically determined PCVs into account. They are able to reduce those systematic errors which could not be eliminated by forming doubledifferences.

\subsection{Baseline comparison with GSOC results}

The reduced-dynamic baseline solutions "N4" with resolved carrier phase ambiguities for the time period of the year 2007 are compared with solutions computed at GSOC. At GSOC, reduced-dynamic baseline solutions are determined with the GHOST package according to a procedure described by Kroes et al. (2005), but with empirical PCVs determined at GSOC taken into account. Due to the independence of the GHOST package and the Bernese GPS Software, it is feasible for the first time to compare independent sets of GRACE baselines based on resolved carrier phase ambiguities.

Figures 11 and 12 show the daily means and the daily STDs of the differences between AIUB and GSOC baseline vectors in the radial, along-track, and cross-track direction 


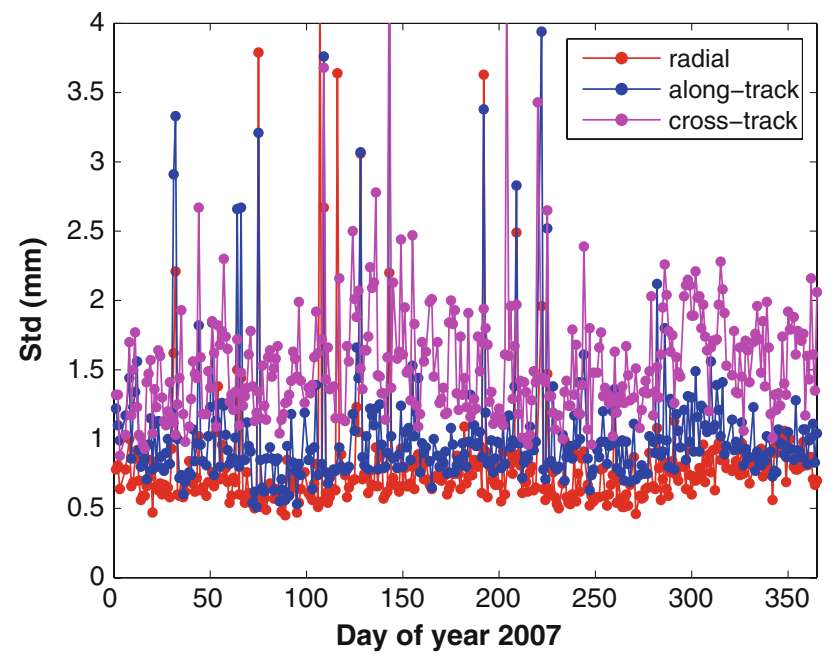

Fig. 12 Daily STDs of differences between AIUB and GSOC baseline vectors based on resolved carrier phase ambiguities

(computed with the help of one of the GRACE A orbits). Figure 11 shows that systematic biases between the two baseline solutions are very small. For the radial and the alongtrack direction they are confined to less than $1 \mathrm{~mm}$, namely to 0.95 and $-0.85 \mathrm{~mm}$. A slightly larger bias of $2.04 \mathrm{~mm}$ is only observed for the cross-track direction, which is probably related to the systematic errors mentioned in Sect. 5.2.3 and 5.3. Even more important are, however, the STDs reported in Fig. 12. They are $0.80 \mathrm{~mm}$ for the radial direction, $1.04 \mathrm{~mm}$ for the along-track direction, and $1.54 \mathrm{~mm}$ for the cross-track direction. The agreement of the radial and the along-track components of the baseline vectors is excellent, whereas a small degradation is again present in the cross-track direction. This is of particular importance in view of the preparation of the TanDEM-X interferometry mission (Krieger et al. 2007)

Within the TanDEM-X mission, the baseline vector between the two satellites has to be determined with an accuracy of $1 \mathrm{~mm}$ (1D RMS, TanDEM-X mission requirements document 2007). In particular, an accurate determination of the line-of-sight component is considered critical for the generation of digital elevation models (DEMs). Errors in the line-of-sight component result in a scene-dependent vertical and, more importantly, lateral shift of the resulting DEM. For a direct matching of overlapping DEMs the lateral errors should be maintained to better than one pixel, which in turn limits the acceptable line-of-sight baseline error to typically $1 \mathrm{~mm}$.

\section{Impact on gravity field recovery}

This section focuses on the importance of empirically determined PCVs for gravity field recovery when using
GPS-derived kinematic LEO positions as pseudo-observations. Since kinematic positions are particularly sensitive to a precise modeling of the antenna phase center location, the propagation of the neglected PCVs via the kinematic positions into the estimated spherical harmonic (SH) coefficients is important to be assessed. For this purpose, three different series of GRACE B kinematic positions covering the entire year 2007 are computed. For the first series, no PCVs are taken into account for the kinematic POD. For the second series, denoted by "N", the empirical PCVs derived from the last iteration of the reduced-dynamic solutions " $\mathrm{N}$ " (cf. Sect. 5.2) are used for the kinematic POD. For the third series, denoted by "K", empirical PCV s are taken into account which are iteratively derived from the residuals stemming from the previous kinematic solution. A large number of ten iterations is performed for the series " $\mathrm{K}$ " to recover the PCVs from the kinematic residuals as completely as possible.

The so-called Celestial Mechanics Approach is used for each set of kinematic positions to compute a static gravity field up to a maximum degree of 90 . For this purpose, the GRACE B kinematic positions are weighted according to the epoch-wise covariance information and serve as pseudoobservations to set up normal equations for the unknown gravity field coefficients in a generalized orbit determination problem (cf. Jäggi et al. 2008) using the gravity field model EGM96 (Lemoine et al. 1997) as a priori information. The resulting normal equations are then accumulated into an annual system, which is eventually inverted to obtain the $\mathrm{SH}$ coefficients and the associated full covariance matrix without applying any regularization.

Figure 13 shows the square-roots of the degree difference variances of the three solutions (based on GPS data) with respect to the superior gravity field model EIGEN-GL04C

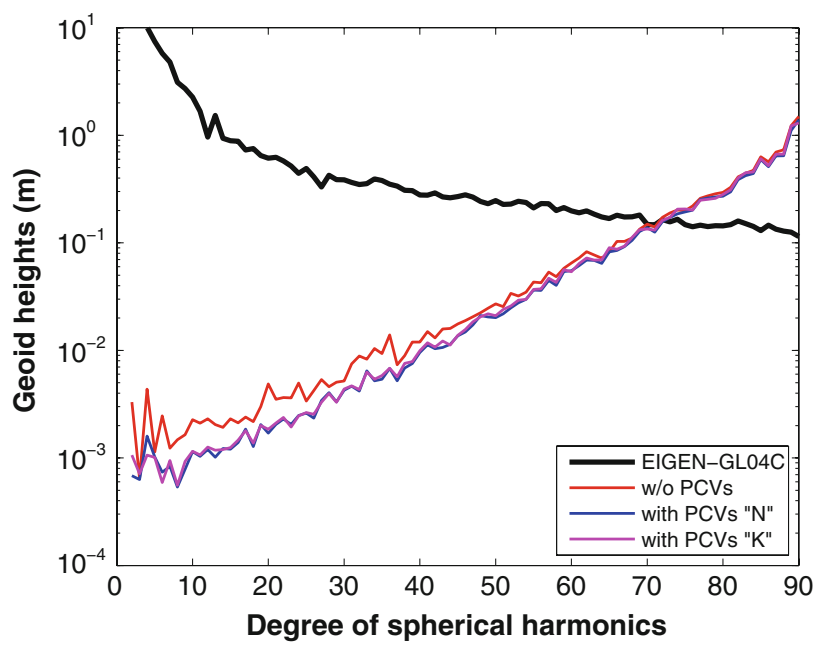

Fig. 13 Square-roots of degree difference variances of annual gravity field recoveries when using different PCV information for the underlying kinematic POD 
(based on K-band data, Förste et al. 2008). It can be easily recognized that there is a significant improvement in the quality of the low degree SH coefficients when taking the empirical PCVs into account. This underlines the importance and necessity to empirically correct for systematic errors in the GPS observations for kinematic POD. For the higher degrees in Fig. 13, however, the improvement is less pronounced. As the patches in the empirical patterns are considerably larger than $1^{\circ}$ (cf. Fig. 6), GPS signals are typically affected by similar systematic errors over a few minutes. Therefore, errors of highest frequency are not introduced into kinematic POD when neglecting PCVs in the modeling of the carrier phase observations.

As the PCVs derived from the reduced-dynamic solutions "N" might potentially be affected by the gravity field model used for the reduced-dynamic orbit determination, the truly independent PCVs derived from the series " $\mathrm{K}$ " are used for comparison. Although it is not possible to extract as much PCV information from the kinematic residuals as from the reduced-dynamic residuals (cf. Sect. 5.2), both patterns show in essence the same performance when using them for gravity field recovery. Small differences are only observed for the very low degrees. This underlines the suitability to use empirical PCVs to further exploit gravity field recovery from GPS hl-SST data.

\section{Conclusions}

The precise knowledge of the phase center location of GNSS antennas is a prerequisite for high-precision LEO orbit determination. Since 5 November 2006, absolute PCO and PCV values are in use for the GNSS transmitter antennas, but consistent values for the LEO receiver antennas are only available since a short time. The PCO and PCV values for the LEO receiver antennas are obtained from ground calibrations of the used antenna/chokering combination and therefore do not reflect the influence of error sources which are additionally encountered in the actual spacecraft environment, e.g., near-field multipath and cross-talk with active GPS occultation antennas. An in-flight calibration of the LEO receiver antennas is thus mandatory.

Two different approaches, the residual approach and the direct approach, were presented for the in-flight determination of empirical corrections to the a priori PCVs of LEO receiver antennas. A simulation study revealed that a complete recovery of PCVs is difficult and that it is governed by a complicated interaction between the recovery approach and the underlying orbit parametrization. The currently used LEO orbit parametrization in the Bernese GPS Software, e.g., asks for an iterative procedure to recover PCVs as completely as possible, PCVs induced by a wrong PCO cannot be recovered at all.
The simulation study showed that the residual approach is limited to recover PCVs of LEO receiver antennas, because GPS-specific parameters such as receiver clock corrections and carrier phase ambiguities partly absorb PCVs (largescale structures). For a fixed alignment of a spacecraft with respect to the orbital frame, e.g., for the two GRACE satellites, PCVs perpendicular to the flight direction are to some extent absorbed by the carrier phase ambiguities. The smallscale structures of PCVs, however, can be well recovered with the residual approach. Deficiencies in the residual approach can be circumvented by the direct approach, where PCVs are directly set up as parameters. As the direct approach is not harmed by the simultaneous estimation of GPS-specific parameters, a more rapid convergence to the final PCV values can be achieved.

Both approaches are limited because LEO positions have to be kept fixed on (degraded) trajectories to derive PCVs iteratively. Only the direct approach has the potential to completely circumvent this problem by a simultaneous estimation of PCV, orbit and clock parameters as well as carrier phase ambiguities. A more dynamic LEO orbit modeling than currently used in the Bernese GPS Software would, however, be a prerequisite to realize this approach without the danger to degrade the "datum" of the resulting LEO trajectories.

The zero-difference reduced-dynamic orbits computed from real GRACE data show a good agreement with independent measurements from other techniques, even if no empirical PCVs are taken into account. The estimated trajectories are confirmed by the SLR technique at a level below $20 \mathrm{~mm}$ RMS error. The inter-satellite distances emerging from our zero-difference GPS analysis are confirmed by the K-band data at a level of about $10 \mathrm{~mm}$ STD for good periods. Taking the empirical PCVs into account, a significant reduction of the K-band residuals to about $6 \mathrm{~mm}$ STD results, demonstrating that unmodeled PCVs induce significant errors into the estimated trajectories. As the structures in the PCVs of the GRACE antennas are considerably larger than $1^{\circ}$, moderate resolutions of about $5^{\circ}$ are sufficient for the needs of POD and further applications.

The analysis of orbits and, in particular, the analysis of directly estimated PCVs revealed unmodeled systematic errors. They primarily affect the cross-track direction of the resulting trajectories and are thus likely to remain undetected, because the cross-track direction is difficult to be validated by independent measurement techniques. We could confirm with both the Bernese GPS Software and the GHOST package that the cross-track component is indeed very sensitive to the settings used for orbit determination, especially in the presence of only partly known PCVs and other mismodeled effects, e.g., related to non-gravitational perturbations. Further investigations are necessary to better confine the origin of these systematic errors. 
The double-difference reduced-dynamic baseline results computed from real GRACE data show that empirical PCVs are indispensable to improve the millimeter precision of ambiguity-fixed space baseline solutions. Although less pronounced than for zero-difference solutions, the K-band validation confirms improvements from $0.9 \mathrm{~mm}$ to about $0.6 \mathrm{~mm}$ STD for good periods. For the first time, the differences between baseline solutions are assessed, which were computed independently with the Bernese GPS Software and the GHOST package. STDs of $0.80 \mathrm{~mm}$ for the radial direction, $1.04 \mathrm{~mm}$ for the along-track direction, and $1.54 \mathrm{~mm}$ for the cross-track direction are obtained, biases for the respective directions are confined to $0.95,-0.85$, and $2.04 \mathrm{~mm}$. Such comparisons are of great importance for the preparation of the GPS data analysis of the upcoming TanDEM-X mission that requires a baseline accuracy of $1 \mathrm{~mm}$ (1D RMS).

The gravity field solutions computed from kinematic GRACE positions underline that empirical PCVs are also indispensable to improve the quality of the estimated low degree SH coefficients of the Earth's gravity field. Depending on the actual LEO receiver antenna pattern, a significant propagation of unmodeled PCVs into the gravity field solution has to be expected. It could be shown that the antenna PCVs contribute significantly to the error budget of GRACE gravity field recovery from GPS hl-SST tracking data. This might be of importance for the current GOCE mission, where the low degree SH coefficients are exclusively determined from GPS data.

Acknowledgments The authors are grateful to the Center for Orbit Determination in Europe (CODE) for using GPS orbit solutions and clock corrections. CODE is a joint venture of the Astronomical Institute of the University of Bern (AIUB, Switzerland), the Federal Office of Topography (swisstopo, Switzerland), the Federal Agency for Cartography and Geodesy (BKG, Germany), and the Institute for Astronomical and Physical Geodesy of the Technische Universität München (IAPG, Germany). The authors are grateful to the Deutsches GeoForschungsZentrum Potsdam (GFZ, Germany), the ILRS, and the GSOC for providing the data for this investigation. The generous financial support provided by the Swiss National Science Foundation and the Institute for Advanced Study (IAS) of the Technische Universität München in the frame of the project "Satellite Geodesy" is gratefully acknowledged. Part of this study was performed in the framework of the GOCE High-level Processing Facility, which is funded by ESA. Finally, we would like to thank R. Schmid and two anonymous reviewers for their constructive comments.

\section{References}

Bertiger WI, Bar-Sever YE, Christensen EJ, Davis ES, Guinn JR, Haines BJ, Ibanez-Meier RW, Jee JR, Lichten SM, Melbourne WG, Muellerschoen RJ, Munson TN, Vigue Y, Wu SC, Yunck TP, Schutz BE, Abusali PAM, Rim HJ, Watkins MM, Willis P (1994) GPS precise tracking of TOPEX/POSEIDON: results and implications. J Geophys Res 99(C12):24449-24464. doi:10. 1029/94JC01171

Bettadpur S (2004) Gravity recovery and climate experiment product specification document. GRACE 327-720 (CSR-GR-03-02),
Rev 4.2, Center for Space Research, The University of Texas at Austin

Beutler G (2005) Methods of celestial mechanics. Springer, Berlin

Bock H, Jäggi A, Švehla D, Beutler G, Hugentobler U, Visser P (2007) Precise orbit determination for the GOCE satellite using GPS. Adv Space Res 39(10):1638-1647. doi:10.1016/j.asr.2007. 02.053

Buckreuss S, Balzer W, Muhlbauer P, Werninghaus R, Pitz W (2003) The TerraSAR-X satellite project. In: Proceedings of IGARSS 2003, vol 5, Toulouse, pp 3096-3098

Dach R, Hugentobler U, Fridez P, Meindl M (eds) (2007) Bernese GPS Software Version 5.0. Documentation, Astronomical Institute, University of Bern, Bern

Dach R, Brockmann E, Schaer S, Beutler G, Meindl M, Prange L, Bock H, Jäggi A, Ostini L (2009) GNSS processing at CODE: status report. J Geod 83(3-4):353-365. doi:10.1007/ s00190-008-0281-2

Dow JM, Neilan RE, Gendt G (2005) The International GPS Service: celebrating the 10th anniversary and looking to the next decade. Adv Space Res 36(3):320-326. doi:10.1016/j.asr.2005.05.125

Drinkwater M, Haagmans R, Muzi D, Popescu A, Floberghagen R, Kern M, Fehringer M (2006) The GOCE gravity mission: ESA's first core explorer. In: Third GOCE User Workshop. 6-8 November 2006. Frascati, Italy, pp 1-7, ESA SP-627

Dunn C, Bertiger W, Bar-Sever Y, Desai S, Haines B, Kuang D, Franklin G, Harris I, Kruizinga G, Meehan T, Nandi S, Nguyen D, Rogstad T, Thomas JB, Tien J, Romans L, Watkins M, Wu SC, Bettadpur S, Kim J (2003) Instrument of GRACE. GPS World 14(2):1728

Förste C, Flechtner F, Schmidt R, Meyer U, Stubenvoll R, Barthelmes F, König R, Neumayer KH, Rothacher M, Reigber C, Biancale R, Bruinsma S, Lemoine JM, Raimondo JC (2005) A new high resolution global gravity field model derived from combination of GRACE and CHAMP mission and altimetry/gravimetry surface gravity data. Geophys Res Abstr 7:04561

Förste C, Schmidt R, Stubenvoll R, Flechtner F, Meyer U, König R, Neumayer H, Biancale R, Lemoine JM, Bruinsma S, Loyer S, Barthelmes F, Esselborn S (2008) The GeoForschungsZentrum Potsdam/Groupe de Recherche de Géodésie Spatiale satellite-only and combined gravity field models: EIGEN-GL04S1 and EIGENGL04C. J Geod 82(6):331-346. doi:10.1007/s00190-007-0183-8

Flechtner F, Schmidt R, Meyer U, Schöne T, Esselborn S, Förste C, Stubenvoll R, Rudenko S, König R, Neumayer KH, Rothacher M (2006) The Benefit of EIGEN Gravity Field Models for Altimetry and vice versa. In: Proceedings of the Symposium on 15 Years of Progress in Radar Altimetry. 13-18 March 2006. Venice, Italy, ESA SP-614

Flechtner F, Bettadpur S, Watkins M, Kruizinga G (2007) GRACE Science Data System Monthly Reports. Available at http://podaac.jpl. nasa.gov/grace/newsletter.html

Fu L-L, Christensen EJ, Yamarone CA, Lefebvre M, Ménard Y, Dorrer M, Escudier P (1994) TOPEX/POSEIDON mission overview. J Geophys Res 99(C12): 24369-24381. doi:10.1029/94JC01761

Haines B, Bar-Sever Y, Bertiger W, Desai S, Willis P (2004) One-centimeter orbit determination for Jason-1: new GPS-based strategies. Mar Geod 27(1-2):299-318. doi:10.1080/01490410490465300

Haines B, Bar-Sever Y, Bertiger W, Desai S, Sibois A, Webb F, Young L (2008) Space-based satellite antenna maps; impact of different satellite antenna maps on LEO \& terrestrial results. 2-6 June 2008. IGS Workshop, Miami, USA

Jacchia LG (1971) Revised static models of the termosphere and exosphere with empirical temperature profiles. SAO Special Report 332

Jäggi A, Hugentobler U, Beutler G (2006) Pseudo-stochastic orbit modeling techniques for low-Earth orbiters. J Geod 80(1):47-60. doi:10.1007/s00190-006-0029-9 
Jäggi A, Hugentobler U, Bock H, Beutler G (2007) Precise orbit determination for GRACE using undifferenced or doubly differenced GPS data. Adv Space Res 39(10):1612-1619. doi:10.1016/j.asr. 2007.03.012

Jäggi A, Beutler G, Prange L, Dach R, Mervart L (2008) Assessment of GPS-only observables for gravity field recovery from GRACE. In: Sideris MG (ed) Observing our changing Earth. Springer, Berlin pp 113-123. doi:10.1007/978-3-540-85426-5_14

Kang Z, Tapley B, Bettadpur S, Ries J, Nagel P, Pastor R (2006) Precise orbit determination for the GRACE mission using only GPS data. J Geod 80(6):322-331. doi:10.1007/s00190-006-0073-5

Koop R, Gruber T, Rummel R (2006) The status of the GOCE highlevel processing facility. In: Third GOCE User Workshop. 6-8 November 2006. Frascati, Italy, pp 195-205, ESA SP-627

Krieger G, Moreira A, Fiedler H, Hajnsek I, Werner M, Younis M, Zink M (2007) TanDEM-X: a satellite formation for high-resolution SAR interferometry. IEEE Trans Geosci Remote Sens 45(11):3317-3341. doi:10.1109/TGRS.2007.900693

Kroes R, Montenbruck O, Bertiger W, Visser PNAM (2005) Precise GRACE baseline determination using GPS. GPS Solut 9(1):2131. doi:10.1007/s10291-004-0123-5

Kroes R (2006) Precise relative positioning of formation flying spacecraft using GPS, vol 61. Publications on Geodesy, Netherlands Geodetic Commission

Lemoine FG, Smith DE, Kunz L, Smith R, Pavlis EC, Pavlis NK, Klosko SM, Chinn DS, Torrence MH, Williamson RG, Cox CM, Rachlin KE, Wang YM, Kenyon SC, Salman R, Trimmer R, Rapp RH, Nerem RS (1997) The development of the NASA GSFC and NIMA joint geopotential model. In: Segawa J, Fujimoto H, Okubo S (eds) IAG symposia: gravity, geoid and marine geodesy. Springer, Berlin, pp 461-469

Luthcke SB, Zelensky NP, Rowlands DD, Lemoine FG, Williams TA (2003) The 1-centimeter orbit: Jason-1 precision orbit determination using GPS, SLR, DORIS, and altimeter data. Mar Geod 26(3):399-421. doi:10.1080/01490410390256727

Montenbruck O, Kroes R (2003) In-flight performance analysis of the CHAMP BlackJack GPS receiver. GPS Solut 7(2):74-86. doi:10. 1007/s10291-003-0055-5

Montenbruck O, van Helleputte T, Kroes R, Gill E (2005) Reduceddynamic orbit determination using GPS code and carrier measurements. Aerosp Sci Technol 9(3):261-271. doi:10.1016/j.ast.2005. 01.003

Montenbruck O, Garcia-Fernandez M, Williams J (2006) Performance comparison of semi-codeless GPS receivers for LEO satellites. GPS Solut 10(4):249-261. doi:10.1007/s10291-006-0025-9

Montenbruck O, Andres Y, Bock H, van Helleputte T, van den Ijssel J, Loiselet M, Marquardt C, Silvestrin P, Visser P, Yoon Y (2008) Tracking and orbit determination performance of the GRAS instrument on MetOp-A. GPS Solut 12(4):289-299. doi:10.1007/s10291-008-0091-2

Montenbruck O, Garcia-Fernandez M, Yoon Y, Schön S, Jäggi A (2009) Antenna phase center calibration for precise positioning of LEO satellites. GPS Solut 13(1):23-34. doi:10.1007/ s10291-008-0094-z

Pail R, Metzler B, Lackner B, Preimesberger T, Höck E, Schuh W-D, Alkathib H, Boxhammer C, Siemes C, Wermuth M (2006) GOCE gravity field analysis in the framework of HPF: operational software system and simulation results. In: 3rd GOCE User Workshop. 6-8 November 2006. Frascati, Italy, ESA SP-627, pp 249-256

Pearlman MR, Degnan JJ, Bosworth JM (2002) The International laser ranging service. Adv Space Res 30(2):135-143. doi:10.1016/ S0273-1177(02)00277-6
Reigber C, Lühr H, Schwintzer P (1998) Status of the CHAMP mission. In: Rummel R, Drewes H, Bosch W, Hornik H (eds) Towards an integrated global geodetic observing system (IGGOS). Springer, Berlin, pp 63-65

Reigber C, Schwintzer P, Neumayer KH, Barthelmes F, König R, Förste C, Balmino G, Biancale R, Lemoine JM, Loyer S, Bruinsma S, Perosanz F, Fayard T (2003) The CHAMP-only earth gravity field model EIGEN-2. Adv Space Res 31(8):1883-1888. doi:10.1016/S0273-1177(03)00162-5

Rockwell International Corporation (1984) GPS interface control document, ICD-GPS-200. Satellite Systems Division

Rothacher M, Schaer S, Mervart L, Beutler G (1995) Determination of antenna phase center variations using GPS data. In: Gendt G, Dick G (eds) Proc 1995 IGS Workshop. GeoForschungsZentrum Potsdam, Potsdam pp 205-220

Schmid R, Rothacher M, Thaller D, Steigenberger P (2005) Absolute phase center corrections of satellite and receiver antennas-impact on global GPS solutions and estimation of azimuthal phase center variations of the satellite antenna. GPS Solut 9(4):283-293. doi:10.1007/s10291-005-0134-X

Schmid R, Steigenberger P, Gendt G, Ge M, Rothacher M (2007) Generation of a consistent absolute phase center correction model for GPS receiver and satellite antennas. J Geod 81(12):781-798. doi:10.1007/s00190-007-0148-y

Švehla D, Rothacher M (2005) Kinematic precise orbit determination for gravity field determination. In: Sansò $\mathrm{F}$ (ed) A window on the future of geodesy. Springer, Berlin pp 181-188. doi:10.1007/ 3-540-27432-4_32

TanDEM-X mission requirements document (2007) TDX-PD-RS0001. Deutsches Zentrum für Luft und Raumfahrt, Oberpfaffenhofen

Tapley BD, Bettadpur S, Watkins M, Reigber C (2004) The gravity recovery and climate experiment: mission overview and early results. Geophys Res Lett 31(9):L09607. doi:10.1029/ 2004GL019920

Teunissen PJG, Kleusberg A (1998) GPS observation equations and positioning concepts. In: Teunissen PJG and Kleusberg A (eds) GPS for Geodesy. Springer, Berlin, pp 187-229

van Helleputte T (2004) User manual for the GHOST orbit determination software, FDS-SUM-3110. Deutsches Zentrum für Luft und Raumfahrt, Oberpfaffenhofen

van Helleputte T, Visser P (2008) GPS based orbit determination using accelerometer data. Aerosp Sci Technol 12(6):478-484. doi:10. 1016/j.ast.2007.11.002

Vallado DA, Finkelman D (2008) A critical assessment of satellite drag and atmospheric density modeling. In: Astrodynamics specialist conference and exhibit. 18-21 August 2008. Honolulu, Hawaii, AIAA 2008-6442

Wu SC, Yunck TP, Thornton CL (1991) Reduced-dynamic technique for precise orbit determination of low Earth satellites. J Guid Control Dyn 14(1):24-30

Wu BH, Chu V, Chen P, Ting T (2005) FORMOSAT-3/COSMIC science mission update. GPS Solut 9(2):111-121. doi:10.1007/ s10291-005-0140-Z

Yoon Y, Eineder M, Yague-Martinez N, Montenbruck O (2009) TerraSAR-X precise trajectory estimation and quality assessment. IEEE Trans Geosci Remote Sens. doi:10.1109/TGRS.2008. 2006983 